\title{
Color Transparency versus Quantum Coherence in Electroproduction of Vector Mesons off Nuclei
}

\author{
B.Z. Kopeliovich ${ }^{1,2,3}$, J. Nemchik ${ }^{4}$, A. Schäfer ${ }^{2}$ and A.V. Tarasov ${ }^{1,2,3}$ \\ ${ }^{1}$ Max-Planck Institut für Kernphysik, Postfach 103980, 69029 Heidelberg, Germany \\ ${ }^{2}$ Institut für Theoretische Physik der Universität, 93040 Regensburg, Germany \\ ${ }^{3}$ Joint Institute for Nuclear Research, Dubna, 141980 Moscow Region, Russia \\ ${ }^{4}$ Institute of Experimental Physics SAV, Watsonova 47, 04353 Kosice, Slovakia
}

\begin{abstract}
So far no theoretical tool for the comprehensive description of exclusive electroproduction of vector mesons off nuclei at medium energies has been developed. We suggest a light-cone QCD formalism which is valid at any energy and incorporates formation effects (color transparency), the coherence length and the gluon shadowing. At medium energies color transparency (CT) and the onset of coherence length (CL) effects are not easily separated. Indeed, although nuclear transparency measured by the HERMES experiment rises with $Q^{2}$, it agrees with predictions of the vector dominance model (VDM) without any CT effects. Our new results and observations are: (i) the good agreement with the VDM found earlier is accidental and related to the specific correlation between $Q^{2}$ and CL for HERMES kinematics; (ii) CT effects are much larger than have been estimated earlier within the two channel approximation. They are even stronger at low than at high energies and can be easily identified by HERMES or at JLab; (iii) gluon shadowing which is important at high energies is calculated and included; (iv) our parameter-free calculations explain well available data for variation of nuclear transparency with virtuality and energy of the photon; (v) predictions for electroproduction of $\rho$ and $\phi$ are provided for future measurements at HERMES and JLab.
\end{abstract}




\section{Introduction: interplay of absorption and shadowing}

\subsection{Color transparency}

The nuclear medium is more transparent for colorless hadronic wave packets than predicted by the Glauber model. One can treat this phenomenon either in the hadronic basis as a results of Gribov's inelastic corrections [1], or in QCD as a result of color screening [2, 3], an effect called color transparency (CT) (see also the review [4]). Although the two approaches are complementary, the latter interpretation is more intuitive and straightforward. Indeed, a point-like colorless object cannot interact with external color fields, therefore its cross section vanishes $\sigma(r) \propto r^{2}$ at $r \rightarrow 0$ [2]. This fact naturally explains the correlation between the cross sections of hadrons and their sizes [5, 6, 7]. When a colorless wave packet propagates through a nucleus, the fluctuations with small size have an enhanced survival probability which leads to a non-exponential attenuation $\propto 1 / L$ [2], where $L$ is the path length in nuclear matter.

Diffractive electroproduction of vector mesons off nuclei is affected by shadowing and absorption which are different phenomena. Final state absorption of the produced meson exists even in the classical probabilistic approach which relates nuclear suppression to the survival probability $W(z, b)$ of the vector meson produced at the point with longitudinal coordinate $z$ and impact parameter $\vec{b}$,

$$
W(z, b)=\exp \left[-\sigma_{i n}^{V N} \int_{z}^{\infty} d z^{\prime} \rho_{A}\left(b, z^{\prime}\right)\right],
$$

where $\rho_{A}(b, z)$ is the nuclear density and $\sigma_{i n}^{V N}$ is the inelastic $V N$ cross section.

Going beyond the VDM one realizes that the diffractive process initiating the production of the vector meson on a bound nucleon is $\gamma^{*} N \rightarrow \bar{q} q N$ (with possible glue). A photon of high virtuality $Q^{2}$ is expected to produce a pair with a small $\sim 1 / Q^{2}$ transverse separation $\llbracket$. The basic idea of CT is that such small size should lead to a vanishing absorption when the colorless $\bar{q} q$ wave packet propagates through the nucleus. However, the pair may evolve in size

\footnotetext{
${ }^{1}$ In fact, the situation is somewhat more complicated. For very asymmetric pairs when the $q$ or $\bar{q}$ carry almost the whole photon momentum, the pair can have a large separation, see Sect. 2.2
} 
during the time of propagation due to transverse motion of the quarks. Besides, the medium filters out large size configurations which have larger absorption cross section円, an effect known as color filtering. Eventually, the resulting distribution amplitude of the $\bar{q} q$ wave packet must be projected onto the wave function of the $V$ meson.

The time scale characterizing the evolution of the $\bar{q} q$ wave packet can be estimated based on the uncertainty principle. One cannot decide whether the ground state $V$ is produced or the next excited state $V^{\prime}$, unless the process lasts longer than the inverse mass difference. In the rest frame of the nucleus this formation time is Lorentz dilated,

$$
t_{f}=\frac{2 \nu}{m_{V}^{2}-m_{V}^{2}},
$$

where $\nu$ is the photon energy.

A rigorous quantum-mechanical description of the pair evolution was suggested in [8] and is based on the light-cone Green function technique. This approach is presented below in Sect. 2.

A complementary description of the same process in the hadronic basis looks quite different 9]. The incident photon may produce different states on a bound nucleon, the $V$ meson ground state or an excited state. Those states propagate through the nucleus experiencing multiple diagonal and off-diagonal diffractive interactions, and eventually the ground state is detected. According to quark-hadron duality we expect these two descriptions to be equivalent. In practice, however, neither of them can be calculated exactly, and therefore each has advantages and shortcomings. For example, electroproduction of light vector mesons on a nucleon cannot be calculated perturbatively without reservations, while in the hadronic basis one can make use of experimental data which include all nonperturbative effects. On the other hand, for excited meson states no data are available for the diagonal and off-diagonal diffractive amplitudes which one can estimate in the quark representation. The two approaches are complementary, they rely on different approximations and their comparison may provide a scale for the theoretical uncertainty involved.

\footnotetext{
${ }^{2}$ Absorption does not mean disappearance or stopping of the quarks. High energy partons usually lose only a very small (energy independent) fraction of their energy, primarily via soft QCD processes. Absorption means color-exchange interaction which switches from the exclusive channel to an inclusive process.
} 


\subsection{Effects of coherence: shadowing of quarks and gluons}

Another phenomenon, shadowing, is also known to cause nuclear suppression. In contrast to final state absorption, it is a pure quantum-mechanical effect which results from destructive interference of the amplitudes for which the interaction takes place on different bound nucleons. It can be interpreted as a competition between the different nucleons participating in the reaction: since the total probability cannot exceed one, each participating nucleon diminishes the chances of others to contribute to the process.

The cross section of photoproduction is very small since it includes the fine structure constant. Applying the Glauber formula one should expect no visible shadowing. However, this is true only at low energies. It has been realized back in the 60s (see the review [10]) that the photon interacts via its hadronic fluctuations. Therefore, if a fluctuation can propagate over a distance comparable or longer than the nuclear radius, it may interact with a large hadronic cross section which causes shadowing. The small probability to create such a fluctuation enters only once, otherwise the fluctuation interacts strongly. Thus, the fluctuation lifetime provides the time scale which controls shadowing. Again, it can be estimated relying on the uncertainty principle and Lorentz time dilation as,

$$
t_{c}=\frac{2 \nu}{Q^{2}+m_{V}^{2}} .
$$

It is usually called coherence time, but we also will use the term coherence length (CL), since light-cone kinematics is assumed, $l_{c}=t_{c}$ (similarly, for formation length $l_{f}=t_{f}$ ). CL is related to the longitudinal momentum transfer $q_{c}=1 / l_{c}$ in $\gamma^{*} N \rightarrow V N$, which controls the interference of the production amplitudes from different nucleons.

Initial state shadowing indeed has been observed in many reactions where no final state absorption is expected, for example in the total photoabsorption cross section on nuclei (see [10]), the inclusive deep-inelastic cross section [11, 12], the total neutrino-nucleus cross section [13], the Drell-Yan reaction of dilepton production [14, 15], etc. In the case of electroproduction of vector mesons off nuclei shadowing and absorption happen with the same cross section which makes it difficult to disentangle the two sources of nuclear suppression. Nevertheless, it is easy 
to identify the difference in the two limiting cases [8] which we illustrate for the example of the vector dominance model (VDM). The first case is the limit of small $l_{c}$, shorter than the mean internucleon spacing $\sim 2 \mathrm{fm}$. In this case only final state absorption matters. The ratio of the quasielastic (or incoherent) $\gamma^{*} A \rightarrow V X$ and $\gamma^{*} N \rightarrow V X$ cross sections, usually called nuclear transparency, reads,

$$
\begin{aligned}
\left.T r_{A}^{i n c}\right|_{l_{c} \ll R_{A}} & \equiv \frac{\sigma_{V}^{\gamma^{*} A}}{A \sigma_{V}^{\gamma^{*} N}}=\frac{1}{A} \int d^{2} b \int_{-\infty}^{\infty} d z \rho_{A}(b, z) \exp \left[-\sigma_{i n}^{V N} \int_{z}^{\infty} d z^{\prime} \rho_{A}\left(b, z^{\prime}\right)\right] \\
& =\frac{1}{A \sigma_{i n}^{V N}} \int d^{2} b\left\{1-\exp \left[-\sigma_{i n}^{V N} T(b)\right]\right\}=\frac{\sigma_{i n}^{V A}}{A \sigma_{i n}^{V N}} .
\end{aligned}
$$

In the limit of long $l_{c}$ it takes a different form,

$$
\left.\operatorname{Tr}_{A}^{i n c}\right|_{l_{c} \gg R_{A}}=\int d^{2} b T_{A}(b) \exp \left[-\sigma_{i n}^{V N} T_{A}(b)\right]
$$

where we assume $\sigma_{e l}^{V N} \ll \sigma_{i n}^{V N}$ for the sake of simplicity. $T_{A}(b)$ is the nuclear thickness function

$$
T_{A}(b)=\int_{-\infty}^{\infty} d z \rho_{A}(b, z) .
$$

The exact expression beyond VDM which interpolates between the two regimes (4) and (5) can be found in [16].

One can see that the $V$ meson attenuates along the whole nucleus thickness in Eq. (5), but only along roughly half of that length in (4). This confirms our conjecture that nuclear shadowing also contributes to (5) increasing suppression. This may be also interpreted as an analog of the quark nuclear shadowing measured in DIS off nuclei, but the absorption effects make this analogy rather shaky.

Gluon shadowing also suppresses electroproduction of $V$ mesons. Different (but equivalent) descriptions of gluon shadowing are known. In the infinite momentum frame of the nucleus it looks like fusion of gluons which overlap in longitudinal direction at small $x$, leading to a reduction of gluon density. In the rest frame of the nucleus the same phenomenon looks as a specific part of Gribov's inelastic corrections [1]. The lowest order inelastic correction related to diffractive dissociation $V N \rightarrow X N$ [17] contains PPR and PPP contributions (in terms of the 
triple-Regge phenomenology, see [18]). The former is related to the quark shadowing already discussed above, while the latter, the triple-Pomeron term, corresponds to gluon shadowing. Indeed, only diffractive gluon radiation can provide the $M_{X}$ dependence $d \sigma_{d d} / d M_{X}^{2} \propto 1 / M_{X}^{2}$ of the diffractive dissociation cross section.

In terms of the light-cone QCD approach the same process is related to the inclusion of higher Fock components, $|\bar{q} q n G\rangle$, containing gluons [19]. Such fluctuations might be quite heavy compared to the simplest $|\bar{q} q\rangle$ fluctuation, therefore, they have a shorter lifetime [20] and need a higher energy to be relevant.

\subsection{Outline of the paper}

In Sect. 2 we present the light-cone (LC) approach to diffractive electroproduction of vector mesons in the rest frame of the nucleon target. The central issue of this approach, the universal interaction cross section for a colorless quark-antiquark dipole and a nucleon, is presented in Sect. 2.1. It cannot be reliably evaluated theoretically and is fitted to the data for the proton structure function in a wide range of $x_{B j}$ and $Q^{2}$.

The LC wave function for a quark-antiquark fluctuation of the virtual photon is presented in Sect. 2.2 for both, free and interacting $\bar{q} q$ pairs. In the latter case we apply the LC Green function approach and introduce into the two-dimensional Schrödinger equation a nonperturbative real LC potential describing the $\bar{q} q$ interaction. The model for the LC wave of a vector meson is described in Sect. 2.3.

As a rigorous test of the model we calculate in Sect. 2.4 the cross section of elastic electroproduction of $\rho$ and $\phi$ mesons off a nucleon target. These parameter-free calculations reproduce both energy and $Q^{2}$ dependence remarkably well, including the absolute normalization. Since we use the nonperturbative LC photon wave function it is legitimate to do calculations down to $Q^{2}=0$. Agreement with data for real photoproduction of $\rho$ and $\phi$ is also good.

Sect. 3 is devoted to incoherent production of vector mesons off nuclei. In Sect. 3.1 the Green function describing propagation of a $\bar{q} q$ in the nuclear medium is modified to incorporate 
absorption. This is done by introducing an imaginary part of the potential into the twodimensional LC Schrödinger equation for the Green function. Different limiting cases of short and long coherence and formation lengths are considered. The central results of the paper is Eq. (52) for the cross section of incoherent vector meson production in the most general case. Numerical calculations and comparison with available data are presented in Sect. 3.2. Nuclear transparency turns out to be a result of a complicated interplay between coherence and formation length effects. Although variation of $l_{c}$ with $Q^{2}$ can mimic CT at medium and low energies, one can map experimental events in $Q^{2}$ and $\nu$ in such a way as to keep $l_{c}=$ const. Unexpectedly, the exact solution found in the present work is very different from the twocoupled-channel approximation of [9] and predicts a much more pronounced effect of CT. This makes it feasible to find a clear signal of CT effects in exclusive production of $\rho$ mesons in the current and planned experiments at HERMES and JLab.

Coherent production of vector mesons off nuclei leaving the nucleus intact is studied in Sect. 4. The formalism described in Sect. 4.1 is simpler than in the case of incoherent production. The detailed calculations and the comparison with data are presented in Sect. 4.2. The effect of CT on the $Q^{2}$ dependence of nuclear transparency at $l_{c}=$ const is weaker than in the case of incoherent production and is difficult to be detected at low energies since the cross section is small. Our results for the differential cross section of coherent production of $\rho$ demonstrate also a weak sensitivity to the CT effects.

Besides CL, there are other effects considered in Sect. 5 which can mimic the phenomenon of CT. First, the standard lowest order inelastic corrections well fixed by available data are known to make the nuclear medium more transparent at higher energies. Since $\nu$ is a rising function of $Q^{2}$ at fixed $l_{c}$, nuclear transparency increases with $Q^{2}$. These corrections are estimated in Sect. 5.1 and the effect is found to be too weak to mock CT. Another source of rising $Q^{2}$ dependence of the nuclear transparency is the finite $\rho$ lifetime which might be important at low energies. This effect evaluated in Sect. 5.2 is also found to be negligibly small.

Exclusive production of vector mesons at high energies is controlled by the small- $x_{B j}$ physics, and gluon shadowing becomes an important phenomenon. It affects the cross section of inco- 
herent vector meson production in a two-fold way. While the production of $V$ on a bound nucleon is suppressed, the nuclear medium becomes more transparent enhancing the survival probability of $\bar{q} q$ wave packets traveling through the nucleus. At the same time, the cross section of coherent production can be only diminished. In Sect. 6 gluon shadowing is calculated and included in the calculations for nuclear transparency.

The results of the paper are summarized and discussed in Sect. 7. An optimistic prognosis for the CT discovery potential of future experiments at HERMES and JLab is made.

\section{Light-cone dipole phenomenology for elastic photopro- duction of vector mesons $\gamma^{*} N \rightarrow V N$}

In the light-cone dipole approach the amplitude of a diffractive process is treated as elastic scattering of a $\bar{q} q$ fluctuation of the incident particle. The elastic amplitude given by convolution of the universal flavor independent dipole cross section for the $\bar{q} q$ interaction with a nucleon, $\sigma_{\bar{q} q}$, which is introduced in [2], and the initial and final wave functions [2]. Thus, the forward production amplitude for the exclusive photo- or electroproduction of vector mesons $\gamma^{*} N \rightarrow$ $V N$ can be represented in the form

$$
\mathcal{M}_{\gamma^{*} N \rightarrow V N}\left(s, Q^{2}\right)=\left\langle V\left|\sigma_{\bar{q} q}^{N}(\vec{\rho}, s)\right| \gamma^{*}\right\rangle=\int_{0}^{1} d \alpha \int d^{2} r \Psi_{V}^{*}(\vec{r}, \alpha) \sigma_{\bar{q} q}(\vec{r}, s) \Psi_{\gamma^{*}}\left(\vec{r}, \alpha, Q^{2}\right)
$$

with the normalization

$$
\left.\frac{d \sigma}{d t}\right|_{t=0}=\frac{|\mathcal{M}|^{2}}{16 \pi}
$$

In order to calculate the photoproduction amplitude one needs to know the following in-

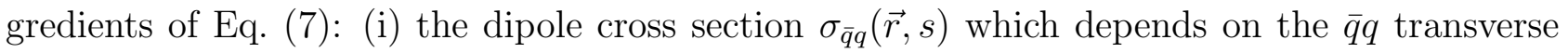
separation $\vec{r}$ and the c.m. energy squared $s$. (ii) The light-cone (LC) wave function of the photon $\Psi_{\gamma^{*}}\left(\vec{r}, \alpha, Q^{2}\right)$ which also depends on the photon virtuality $Q^{2}$ and the relative share $\alpha$ of the photon momentum carried by the quark. (iii) The LC wave function $\Psi_{V}(\vec{r}, \alpha)$ of the vector meson. They are presented in the following sections. 
Note that in the LC formalism the photon and meson wave functions contain also higher Fock states $|\bar{q} q\rangle,|\bar{q} q G\rangle,|\bar{q} q 2 G\rangle$, etc. Should one add their contribution to Eq. (8)? A word of caution is in order. The energy dependence of the total cross section including the dipole one as is given in Eq. (7), originates from inelastic collisions with gluon bremsstrahlung, a process related to the forward elastic amplitude via unitarity. Those inelastic collisions also can be described in terms of the Fock components containing gluons. Thus, one would double count if both the energy dependent dipole cross section and the higher Fock states were included. Either one should rely upon the Fock state decomposition treating interaction of each of them as energy independent, or one should restrict ones consideration to the lowest $|\bar{q} q\rangle$ component, but implicitly incorporate the effects of higher Fock states into the energy dependence of the dipole cross section $\sigma_{\bar{q} q}$. We stand with the latter approach in the present paper.

However, as for nuclear targets, one must explicitly include into ones consideration the higher Fock states because their eikonalization leads to gluon shadowing. We come back to this problem in Sect. 6.

\subsection{Phenomenological dipole cross section}

The cross section $\sigma_{\bar{q} q}(\vec{r}, s)$ for the interaction of a $\bar{q} q$ dipole of transverse separation $\vec{r}$ with a nucleon, first introduced in [2], is a flavor independent universal function of $\vec{r}$ and energy. It allows to describe in a uniform way various high energy processes. This cross section still cannot be predicted reliably because of poorly known higher order pQCD corrections and nonperturbative effects. However, it is known to vanish quadratically $\sigma_{\bar{q} q}(r, s) \propto r^{2}$ as $r \rightarrow 0$ due to color screening, a property usually called color transparency. On the other hand, one may expect the dipole cross section to level off at large separations. This may happen if the quark density in the proton already saturates in the $x_{B j}$ range of HERA [21, 22]. Whether this was already observed at HERA in the small $x_{B j}$ domain is difficult to say. One can fit the data perfectly either assuming saturation [22], or with the pure DGLAP evolution. One can interpret the leveling off of the dipole cross section not only in terms of saturated parton 
density (these two are not identical). Another scenario relates the flat behavior of $\sigma_{\bar{q} q}(r, s)$ at large $r$ to the averaged gluon propagation length $r_{0}$. For $r^{2} \gg r_{0}^{2}$ one arrives in this case at the additive quark model: the dipole cross section is a sum of quark-quark cross sections, i.e. the $\sigma_{\bar{q} q}(r)$ levels off at large separations.

At small separations the dipole cross section should be a function of $r$ and $x_{B j} \sim 1 /\left(r^{2} s\right)$ to reproduce Bjorken scaling. A corresponding simple and popular parameterization has been suggested in [22]. It well describes data for DIS at small $x$ and medium and high $Q^{2}$. However, at small $Q^{2}$ it cannot be correct since it predicts energy independent hadronic cross sections. Besides, $x_{B j}$ is not any more a proper variable at small $Q^{2}$ and should be replaced by energy. Since we want our approach to be valid down to the limit of real photoproduction, we choose the parametrization suggested in [23] which is similar to one in [22], but contains an explicit dependence on energy,

$$
\sigma_{\bar{q} q}(r, s)=\sigma_{0}(s)\left[1-e^{-r^{2} / r_{0}^{2}(s)}\right]
$$

It correctly reproduces the hadronic cross sections for the choice

$$
\begin{gathered}
\sigma_{0}(s)=\sigma_{\text {tot }}^{\pi p}(s)\left[1+\frac{3}{8} \frac{r_{0}^{2}(s)}{\left\langle r_{c h}^{2}\right\rangle}\right] \mathrm{mb} \\
r_{0}(s)=0.88\left(\frac{s}{s_{0}}\right)^{-0.14} \mathrm{fm} .
\end{gathered}
$$

Here $\left\langle r_{c h}^{2}\right\rangle=0.44 \mathrm{fm}^{2}$ is the mean pion charge radius squared; $s_{0}=1000 \mathrm{GeV}^{2}$. The cross section $\sigma_{\text {tot }}^{\pi p}(s)$ was fitted to data in [24, 25],

$$
\sigma_{\text {tot }}^{\pi p}(s)=23.6\left(\frac{s}{s_{0}}\right)^{0.079}+0.032\left(\frac{s}{s_{0}}\right)^{-0.45} \mathrm{mb} \text {. }
$$

It represents the Pomeron and Reggeon parts corresponding to exchange in of gluons and $\bar{q} q$, respectively. Only the former has been used in the dipole cross section Eq. (9) to fit the data for the proton structure function at small $x_{B j}$. Unfortunately, the Reggeon part of the dipole cross section is poorly known. To the best of our knowledge no phenomenology of it has been developed so far. The energy dependence of the Reggeon dipole cross section at small $Q^{2}$ (or $x$-dependence at high $\left.Q^{2}\right)$ dictated by Regge phenomenology is approximately $\propto 1 / \sqrt{s}(\propto \sqrt{x})$. 
Thus, we can expect $Q^{2}$ independence of the the exponent in the second term on the r.h.s of Eq. (12). The $r$ dependence of this term in the dipole cross section is less known. In order to reproduce Bjorken scaling we must assume that the Regge term vanishes at $r \rightarrow 0$ in the same way as the Pomeron part of the cross section, $\sigma_{R}(r, s) \propto r^{2}$. For the sake of simplicity we therefore assume the same parameterization as is used for the Pomeron part, Eq. (9)). Then, one can just incorporate the Reggeon term into $\sigma_{0}(s)$ as is done in Eq. (12).

Incorporating Reggeons into the LC dipole formalism for nuclear shadowing one should be careful with the treatment of multiple interaction terms which have a nonplanar nature [26]. The Reggeon exchange described by planar graphs should not participate in the multiple-scattering expansion of the eikonal exponential.

The dipole cross section Eqs.(99) - (12) provides the imaginary part of the elastic amplitude. It is known, however, that the energy dependence of the total cross section generates also a real part [27,

$$
\sigma_{\bar{q} q}(r, s) \Rightarrow\left(1-i \frac{\pi}{2} \frac{\partial}{\partial \ln (s)}\right) \sigma_{\bar{q} q}(r, s)
$$

The energy dependence of the dipole cross section Eq. (99) is rather steep at small $r$ leading to a large real part which should not be neglected. For instance, the photoproduction amplitude of $\gamma N \rightarrow J / \Psi N$ rises $\propto s^{0.2}$ and the real-to-imaginary part ratio is over $30 \%$. At medium energies also the Reggeon contribution to electroproduction of light mesons contributes to the real part of the elastic amplitude. The replacement Eq. (13) takes care of it as well, and we use this form everywhere in what follows, unless specified otherwise.

Note that the improvement compared to [22] at large separations leads to a worse description of the short-distance part of the dipole cross section which is responsible for the behavior of the proton structure function at large $Q^{2}$. To satisfy Bjorken scaling the dipole cross section at small $r$ must be a function of the product $s r$ which is not the case for the parametrization in Eq. (9). Indeed, the form of Eq. (9) successfully describes data for DIS at small $x$ only up to $Q^{2} \approx 10 \mathrm{GeV}^{2}$, and does a poor job at larger values of $Q^{2}$. Nevertheless, this interval of $Q^{2}$ is sufficient for the purpose of the present paper which is focused on production of light vector 
mesons at small and moderate $Q^{2} \lesssim 10 \mathrm{GeV}^{2}$.

\subsection{The $\bar{q} q$ wave function of the photon}

The perturbative distribution amplitude ("wave function") of the $\bar{q} q$ Fock component of the photon is well known [28, 29, 30], and for transversely (T) and longitudinally (L) polarized photons it has the form,

$$
\Psi_{\bar{q} q}^{T, L}(\vec{r}, \alpha)=\frac{\sqrt{N_{C} \alpha_{e m}}}{2 \pi} Z_{q} \bar{\chi} \hat{O}^{T, L} \chi K_{0}(\epsilon r)
$$

where $\chi$ and $\bar{\chi}$ are the spinors of the quark and antiquark, respectively; $Z_{q}$ is the quark charge; $N_{C}=3$ is the number of colors. $K_{0}(\epsilon r)$ is a modified Bessel function with

$$
\epsilon^{2}=\alpha(1-\alpha) Q^{2}+m_{q}^{2}
$$

where $m_{q}$ is the quark mass, and $\alpha$ is the fraction of the LC momentum of the photon carried by the quark. The operators $\widehat{O}^{T, L}$ read,

$$
\begin{gathered}
\widehat{O}^{T}=m_{q} \vec{\sigma} \cdot \vec{e}+i(1-2 \alpha)(\vec{\sigma} \cdot \vec{n})\left(\vec{e} \cdot \vec{\nabla}_{r}\right)+(\vec{\sigma} \times \vec{e}) \cdot \vec{\nabla}_{r} \\
\widehat{O}^{L}=2 Q \alpha(1-\alpha)(\vec{\sigma} \cdot \vec{n}) .
\end{gathered}
$$

Here $\vec{\nabla}_{r}$ acts on transverse coordinate $\vec{r} ; \vec{e}$ is the polarization vector of the photon and $\vec{n}$ is a unit vector parallel to the photon momentum.

The transverse $\bar{q} q$ separation is controlled by the distribution amplitude Eq. (14) with the mean value,

$$
\langle r\rangle \sim \frac{1}{\epsilon}=\frac{1}{\sqrt{Q^{2} \alpha(1-\alpha)+m_{q}^{2}}} .
$$

In pQCD the quarks are treated as free, and one may wonder why they do not fly apart but form a wave packet of finite size. It is interference of $\bar{q} q$ waves produced at different points which keeps the transverse separation finite. To reach a large separation the $\bar{q} q$ pair must be produced sufficiently long in advance, longer than the coherence time Eq. (3), such that fluctuations loose coherence. Treating the coherence time as lifetime of the fluctuation, one can also say that the fluctuation does not have enough time to fly apart. 
For very asymmetric $\bar{q} q$ pairs with $\alpha$ or $(1-\alpha) \lesssim m_{q}^{2} / Q^{2}$ the mean transverse separation $\langle r\rangle \sim 1 / m_{q}$ becomes huge since one must use current quark masses within pQCD. A popular recipe to fix this problem is to introduce an effective quark mass $m_{\text {eff }} \sim \Lambda_{Q C D}$ which should represent the nonperturbative interaction effects between $q$ and $\bar{q}$. It is more consistent, however, and straightforward to introduce this interaction explicitly. The corresponding phenomenology based on the light-cone Green function approach has been developed in [23].

The Green function $G_{\bar{q} q}\left(z_{1}, \vec{r}_{1} ; z_{2}, \vec{r}_{2}\right)$ describes the propagation of an interacting $\bar{q} q$ pair between points with longitudinal coordinates $z_{1}$ and $z_{2}$ and with initial and final separations $\vec{r}_{1}$ and $\vec{r}_{2}$. This Green function satisfies the two-dimensional Schrödinger equation,

$$
i \frac{d}{d z_{2}} G_{\bar{q} q}\left(z_{1}, \vec{r}_{1} ; z_{2}, \vec{r}_{2}\right)=\left[\frac{\epsilon^{2}-\Delta_{r_{2}}}{2 \nu \alpha(1-\alpha)}+V_{\bar{q} q}\left(z_{2}, \vec{r}_{2}, \alpha\right)\right] G_{\bar{q} q}\left(z_{1}, \vec{r}_{1} ; z_{2}, \vec{r}_{2}\right) .
$$

Here $\nu$ is the photon energy. The Laplacian $\Delta_{r}$ acts on the coordinate $r$.

The imaginary part of the LC potential $V_{\bar{q} q}\left(z_{2}, \vec{r}_{2}, \alpha\right)$ in (19) is responsible for attenuation of the $\bar{q} q$ in the medium, while the real part represents the interaction between the $q$ and $\bar{q}$. This potential is supposed to provide the correct LC wave functions of vector mesons. For the sake of simplicity we use the oscillator form of the potential,

$$
\operatorname{Re} V_{\bar{q} q}\left(z_{2}, \vec{r}_{2}, \alpha\right)=\frac{a^{4}(\alpha) \vec{r}_{2}^{2}}{2 \nu \alpha(1-\alpha)}
$$

which leads to a Gaussian $r$-dependence of the LC wave function of the meson ground state. The shape of the function $a(\alpha)$ will be discussed in the next section.

In this case equation (19) has an analytical solution, the harmonic oscillator Green function 31,

$$
\begin{array}{r}
G_{\bar{q} q}\left(z_{1}, \vec{r}_{1} ; z_{2}, \vec{r}_{2}\right)=\frac{a^{2}(\alpha)}{2 \pi i \sin (\omega \Delta z)} \exp \left\{\frac{i a^{2}(\alpha)}{\sin (\omega \Delta z)}\left[\left(r_{1}^{2}+r_{2}^{2}\right) \cos (\omega \Delta z)-2 \vec{r}_{1} \cdot \vec{r}_{2}\right]\right\} \\
\times \exp \left[-\frac{i \epsilon^{2} \Delta z}{2 \nu \alpha(1-\alpha)}\right],
\end{array}
$$

where $\Delta z=z_{2}-z_{1}$ and

$$
\omega=\frac{a^{2}(\alpha)}{\nu \alpha(1-\alpha)} .
$$


The boundary condition is $\left.G_{\bar{q} q}\left(z_{1}, \vec{r}_{1} ; z_{2}, \vec{r}_{2}\right)\right|_{z_{2}=z_{1}}=\delta^{2}\left(\vec{r}_{1}-\vec{r}_{2}\right)$.

The probability amplitude to find the $\bar{q} q$ fluctuation of a photon at the point $z_{2}$ with separation $\vec{r}$ is given by an integral over the point $z_{1}$ where the $\bar{q} q$ is created by the photon with initial separation zero,

$$
\Psi_{\bar{q} q}^{T, L}(\vec{r}, \alpha)=\left.\frac{i Z_{q} \sqrt{\alpha_{e m}}}{4 \pi E \alpha(1-\alpha)} \int_{-\infty}^{z_{2}} d z_{1}\left(\bar{\chi} \widehat{O}^{T, L} \chi\right) G_{\bar{q} q}\left(z_{1}, \vec{r}_{1} ; z_{2}, \vec{r}\right)\right|_{r_{1}=0} .
$$

The operators $\widehat{O}^{T, L}$ are defined in Eqs. (16) and (17). Here they act on the coordinate $\vec{r}_{1}$.

If we write the transverse part as

$$
\bar{\chi} \widehat{O}^{T} \chi=A+\vec{B} \cdot \vec{\nabla}_{r_{1}},
$$

then the distribution functions read,

$$
\begin{gathered}
\Psi_{\bar{q} q}^{T}(\vec{r}, \alpha)=Z_{q} \sqrt{\alpha_{e m}}\left[A \Phi_{0}(\epsilon, r, \lambda)+\vec{B} \vec{\Phi}_{1}(\epsilon, r, \lambda)\right], \\
\Psi_{\bar{q} q}^{L}(\vec{r}, \alpha)=2 Z_{q} \sqrt{\alpha_{e m}} Q \alpha(1-\alpha) \bar{\chi} \vec{\sigma} \cdot \vec{n} \chi \Phi_{0}(\epsilon, r, \lambda),
\end{gathered}
$$

where

$$
\lambda=\frac{2 a^{2}(\alpha)}{\epsilon^{2}} .
$$

The functions $\Phi_{0,1}$ in Eqs. (25) and (26) are defined as

$$
\begin{gathered}
\Phi_{0}(\epsilon, r, \lambda)=\frac{1}{4 \pi} \int_{0}^{\infty} d t \frac{\lambda}{\operatorname{sh}(\lambda t)} \exp \left[-\frac{\lambda \epsilon^{2} r^{2}}{4} \operatorname{cth}(\lambda t)-t\right], \\
\vec{\Phi}_{1}(\epsilon, r, \lambda)=\frac{\epsilon^{2} \vec{r}}{8 \pi} \int_{0}^{\infty} d t\left[\frac{\lambda}{\operatorname{sh}(\lambda t)}\right]^{2} \exp \left[-\frac{\lambda \epsilon^{2} r^{2}}{4} \operatorname{cth}(\lambda t)-t\right] .
\end{gathered}
$$

Note that the $\bar{q}-q$ interaction enters Eqs. (25) and (26) via the parameter $\lambda$ defined in (27). In the limit of vanishing interaction $\lambda \rightarrow 0$ (i.e. $Q^{2} \rightarrow \infty, \alpha$ is fixed, $\alpha \neq 0$ or 1 ) Eqs. (25) - (26) produce the perturbative expressions of Eq. (14).

With the choice $a^{2}(\alpha) \propto \alpha(1-\alpha)$ the end-point behavior of the mean square interquark separation $\left\langle r^{2}\right\rangle \propto 1 / \alpha(1-\alpha)$ contradicts the idea of confinement. Following [23] we fix this problem via a simple modification of the LC potential,

$$
a^{2}(\alpha)=a_{0}^{2}+4 a_{1}^{2} \alpha(1-\alpha)
$$


The parameters $a_{0}$ and $a_{1}$ were adjusted in [23] to data on total photoabsorption cross section [32, 33], diffractive photon dissociation and shadowing in nuclear photoabsorption reaction. The results of our calculations vary within only $1 \%$ when $a_{0}$ and $a_{1}$ satisfy the relation,

$$
\begin{aligned}
& a_{0}^{2}=v^{1.15}(0.112)^{2} \mathrm{GeV}^{2} \\
& a_{1}^{2}=(1-v)^{1.15}(0.165)^{2} \mathrm{GeV}^{2},
\end{aligned}
$$

where $v$ takes any value $0<v<1$. In view of this insensitivity of the observables we fix the parameters at $v=1 / 2$. We checked that this choice does not affect our results beyond a few percent uncertainty.

\subsection{The meson wave function}

To describe electroproduction reactions it is natural to work in the infinite momentum frame of the virtual photon and use the LC variables for the $\bar{q} q$ pair, the transverse separation $\vec{r}$ and the fraction $\alpha=p_{q}^{+} / p_{V}^{+}$of the total LC momentum carried by the quark. The wave functions of light vector mesons are poorly known both in the rest and infinite momentum frames. A popular prescription [34] is to apply the Lorentz boost to the rest frame wave function assumed to be Gaussian which leads to radial parts of transversely and longitudinally polarized mesons in the form,

$$
\Phi_{V}^{T, L}(\vec{r}, \alpha)=C^{T, L} \alpha(1-\alpha) f(\alpha) \exp \left[-\frac{\alpha(1-\alpha) \vec{r}^{2}}{2 R^{2}}\right]
$$

with a normalization defined below, and

$$
f(\alpha)=\exp \left[-\frac{m_{q}^{2} R^{2}}{2 \alpha(1-\alpha)}\right] .
$$

This procedure is ill motivated since the $\bar{q} q$ are not classical particles. As a result of the boost to the infinite momentum frame many new Fock components are created. Nevertheless, a detailed analysis of this problem [35] leads to the same form (32) which we use in what follows with the parameters from 36, $R=0.59 \mathrm{fm}$ and $m_{q}=0.15 \mathrm{GeV}$. 
We assume that the distribution amplitude of $\bar{q} q$ fluctuations for the vector meson and for the photon have a similar structure [36]. Then in analogy to Eqs. (25) - (26),

$$
\begin{aligned}
\Psi_{V}^{T}(\vec{r}, \alpha) & =(A+\vec{B} \cdot \vec{\nabla}) \Phi_{V}^{T}(r, \alpha) ; \\
\Psi_{V}^{L}(\vec{r}, \alpha) & =2 m_{V} \alpha(1-\alpha)(\bar{\chi} \vec{\sigma} \cdot \vec{n} \chi) \Phi_{V}^{L}(r, \alpha) .
\end{aligned}
$$

Correspondingly, the normalization conditions for the transverse and longitudinal vector meson wave functions read,

$$
\begin{gathered}
N_{C} \int d^{2} r \int d \alpha\left\{m_{q}^{2}\left|\Phi_{V}^{T}(\vec{r}, \alpha)\right|^{2}+\left[\alpha^{2}+(1-\alpha)^{2}\right]\left|\partial_{r} \Phi_{V}^{T}(\vec{r}, \alpha)\right|^{2}\right\}=1 \\
4 N_{C} \int d^{2} r \int d \alpha \alpha^{2}(1-\alpha)^{2} m_{V}^{2}\left|\Phi_{V}^{L}(\vec{r}, \alpha)\right|^{2}=1 .
\end{gathered}
$$

\subsection{Cross section on a nucleon, comparison with data}

Now we are in the position to calculate the forward production amplitude $\gamma^{*} N \rightarrow V N$ for transverse and longitudinal photons and vector mesons using the nonperturbative photon wave functions Eqs. (25), (26) and for the vector meson Eqs. (34), (35). We verify the LC approach by comparing with data for nucleon target. This is a rigorous test since we have no free parameters.

The forward scattering amplitude reads,

$$
\begin{aligned}
\left.\mathcal{M}_{\gamma^{*} N \rightarrow V N}^{T}\left(s, Q^{2}\right)\right|_{t=0} & =N_{C} Z_{q} \sqrt{\alpha_{e m}} \int d^{2} r \sigma_{\bar{q} q}(\vec{r}, s) \int_{0}^{1} d \alpha\left\{m_{q}^{2} \Phi_{0}(\epsilon, \vec{r}, \lambda) \Psi_{V}^{T}(\vec{r}, \alpha)\right. \\
& \left.+\left[\alpha^{2}+(1-\alpha)^{2}\right] \vec{\Phi}_{1}(\epsilon, \vec{r}, \lambda) \cdot \vec{\nabla}_{r} \Psi_{V}^{T}(\vec{r}, \alpha)\right\} \\
\left.\mathcal{M}_{\gamma^{*} N \rightarrow V N}^{L}\left(s, Q^{2}\right)\right|_{t=0} & =4 N_{C} Z_{q} \sqrt{\alpha_{e m}} m_{V} Q \int d^{2} r \sigma_{\bar{q} q}(\vec{r}, s) \\
& \times \int_{0}^{1} d \alpha \alpha^{2}(1-\alpha)^{2} \Phi_{0}(\epsilon, \vec{r}, \lambda) \Psi_{V}^{L}(\vec{r}, \alpha) .
\end{aligned}
$$

These amplitudes are normalized as $\left|\mathcal{M}^{T, L}\right|^{2}=16 \pi d \sigma_{N}^{T, L} /\left.d t\right|_{t=0}$. We include the real part of the amplitude according to the prescription described in Sect. 2.1. In what follows we calculate the cross sections $\sigma=\sigma^{T}+\epsilon \sigma^{L}$ assuming that the photon polarization is $\epsilon=1$. 
Now we can check the absolute value of the predicted cross section by comparing with data for elastic electroproduction $\gamma^{*} p \rightarrow V p$ for $\rho$ and $\phi$ mesons. Unfortunately, data are available only for the cross section integrated over $t$,

$$
\sigma^{T, L}\left(\gamma^{*} N \rightarrow V N\right)=\frac{\left|\mathcal{M}^{T, L}\right|^{2}}{16 \pi B_{\gamma^{*} N}}
$$

where the $t$-slope of the differential cross section which cannot be properly predicted by the approach under consideration. Our strategy is to predict the numerator in (40), and compare with data for the cross section and the slope.

Our predictions are plotted in Fig. 1 together with the data on the $Q^{2}$ dependence of the cross section from NMC, H1 and ZEUS [37, 38, 39, 40]).

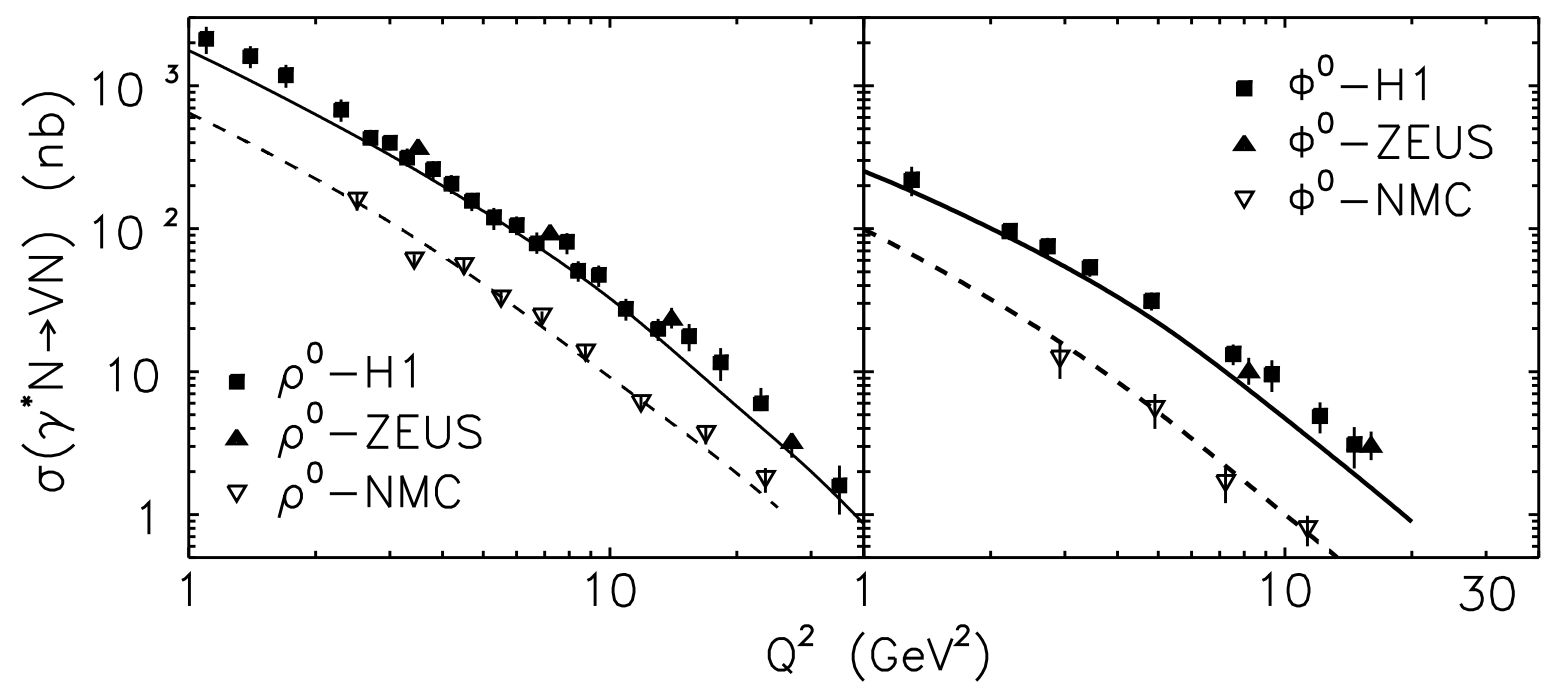

Figure 1: $Q^{2}$ - dependence of the cross section for the reactions $\gamma^{*} p \rightarrow \rho p$ (left) and $\gamma^{*} p \rightarrow \phi p$ (right). The dashed and solid curves are compared with data at $W=15 \mathrm{GeV}$ [37] and at $75 \mathrm{GeV}$ (38, 39] for $\rho$ and [40] for $\phi)$, respectively.

We use the $Q^{2}$ dependent slope of the differential cross section $d \sigma\left(\gamma^{*} N \rightarrow V N\right) / d t \propto$ $\exp \left[B_{\gamma^{*} N}^{V}\left(Q^{2}\right) t\right]$ parametrized as 41 ,

$$
B_{\gamma^{*} N}\left(s, Q^{2}\right)=\beta_{0}^{V}(s)+\frac{\beta_{1}^{V}(s)}{Q^{2}+m_{V}^{2}}-\frac{1}{2} \ln \left(\frac{Q^{2}+m_{V}^{2}}{m_{V}^{2}}\right) .
$$


A fit to the data [37, 42] from fixed target experiments for the $Q^{2}$ dependent slope in $\rho$ production give the parameters for $W \approx 10-15 \mathrm{GeV}, \beta_{0}^{\rho}=(6.2 \pm 0.2) \mathrm{GeV}^{-2}, \beta_{1}^{\rho}=1.5 \pm 0.2$. Using data from HERA [38, 39, 43, 44, 45, 46, 47] for $\rho$ production at $W=75 \mathrm{GeV}$ we get, $\beta_{0}^{\rho}=(7.1 \pm 0.1) \mathrm{GeV}^{-2}, \beta_{1}^{\rho}=2.0 \pm 0.1$.

Repeating the same analysis for $\phi$ production we get at $W \approx 10-15 \mathrm{GeV}$ from data [37, 48], $\beta_{0}^{\phi}=(5.9 \pm 0.1) \mathrm{GeV}^{-2}, \beta_{1}^{\phi}=0.5 \pm 0.1$. Data from HERA 49, 40 give, $\beta_{0}^{\phi}=(6.7 \pm 0.2) \mathrm{GeV}^{-2}$, $\beta_{1}^{\phi}=1.0 \pm 0.1$. For calculations shown in Fig. 1 we use the central values of these parameters.

Our approach which includes the effects of the nonperturbative interaction between the $q$ and $\bar{q}$ in the photon fluctuation is designed to describe the low $Q^{2}$ region as well. To test it we compare with data [50, 51, 44, 45, 52 for the energy dependence of the cross section of real $\rho$ photoproduction in Fig. 2. We use the energy dependent slope parameter, $B_{\gamma N}^{\rho}=$ $B_{0}^{\rho}+2 \alpha^{\prime} \ln \left(s / s_{0}\right)$ with $\alpha^{\prime}=0.25 \mathrm{GeV}^{-2}$ and $B_{0}^{\rho}=7.6 \mathrm{GeV}^{-2}, s_{0}=20 \mathrm{GeV}^{2}$ fitted to data [53, 50, 43, 45, 46]. The Pomeron part of the dipole cross section depicted by the dashed curve in Fig. 2 cannot explain the data at low energies, $W \lesssim 15 \mathrm{GeV}$, while the addition of the Regge term (solid curve) leads to a good agreement for all energies. We also found a good agreement with data for real photoproduction of $\phi$, but skip the comparison since there are very few data points.

The normalization of the cross section and its energy and $Q^{2}$ dependence are remarkably well reproduced in Figs. 1 - 2. This is an important achievement since the absolute normalization is usually much more difficult to reproduce than nuclear effects. For instance, the similar, but simplified calculations in [8] underestimate the $J / \Psi$ photoproduction cross section on protons by an order of magnitude.

As a cross-check for the choice of the $\rho^{0}$ wave function in Eqs. (32) and (30) we also calculated the total $\rho^{0}$-nucleon cross section, which is usually expected to be roughly similar to the pion-nucleon cross section $\sigma_{\text {tot }}^{\pi N} \sim 25 \mathrm{mb}$. The $\rho$-nucleon total cross section has the form,

$$
\sigma_{\text {tot }}^{\rho N}=N_{C} \int d^{2} r \int d \alpha\left\{m_{q}^{2}\left|\Phi_{V}^{T}(\vec{r}, \alpha)\right|^{2}+\left[\alpha^{2}+(1-\alpha)^{2}\right]\left|\partial_{r} \Phi_{V}^{T}(\vec{r}, \alpha)\right|^{2}\right\} \sigma_{\bar{q} q}(\vec{r}, s)
$$

We calculated $\sigma_{\text {tot }}^{\rho N}$ with the $\rho$ meson wave function in the form Eq. (32) with the parameters 


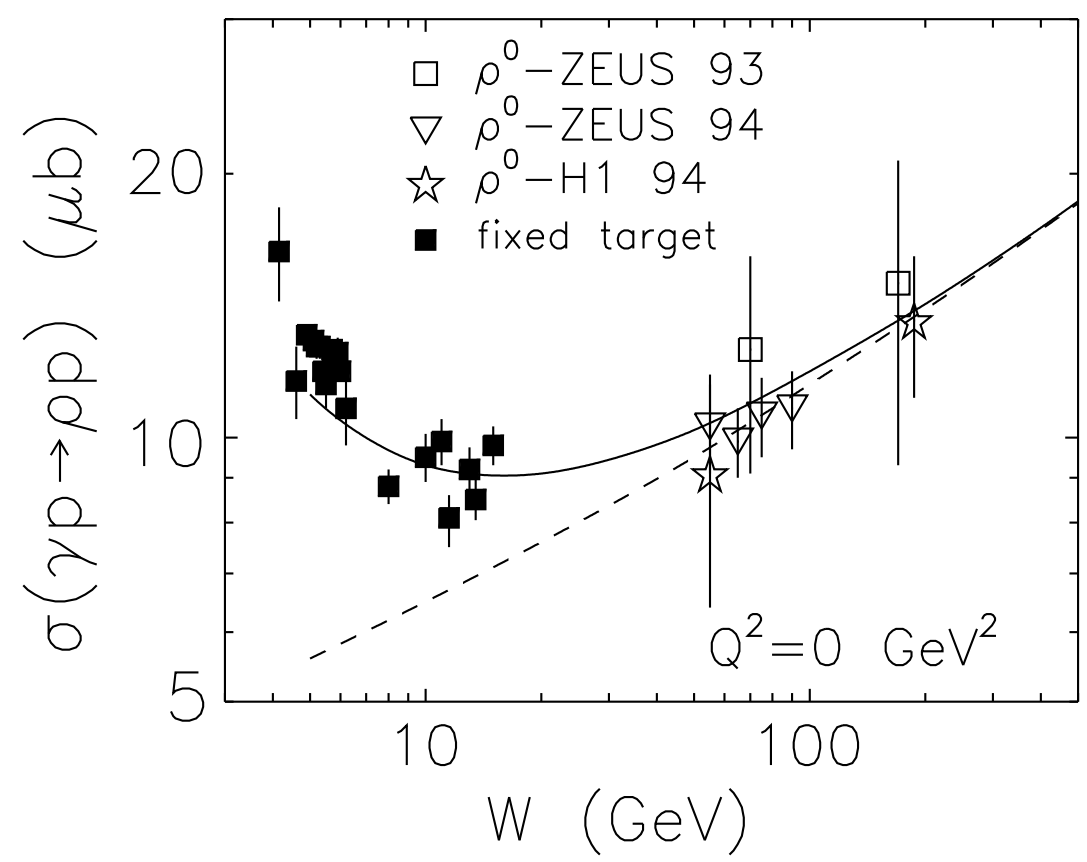

Figure 2: Energy dependence of the real photoproduction cross section on a nucleon, $\gamma p \rightarrow \rho^{0} p$. Our results (solid curve) are compared with data from the fixed target [50, 51], and collider HERA H1 [44] and ZEUS [45, 52] experiments. The dashed curve contains only the gluonic exchange in the t-channel.

described in the Sect. 2.3. For the dipole cross section we adopt the KST parameterization (9) which is designed to describe low- $Q^{2}$ data. Then, at $\nu=100 \mathrm{GeV}$ we obtain $\sigma_{t o t}^{\rho N}=27 \mathrm{mb}$ which is quite a reasonable number.

\section{Incoherent production of vector mesons off nuclei}

In diffractive incoherent (quasielastic) production of vector mesons off nuclei, $\gamma^{*} A \rightarrow V X$, one sums over all final states of the target nucleus except those which contain particle (pion) creation. The observable usually studied experimentally is nuclear transparency defined as

$$
\operatorname{Tr}_{A}^{i n c}=\frac{\sigma_{\gamma^{*} A \rightarrow V X}^{i n c}}{A \sigma_{\gamma^{*} N \rightarrow V N}} .
$$


The $t$-slope of the differential quasielastic cross section is the same as on a nucleon target. Therefore, instead of integrated cross sections one can also use the forward differential cross sections Eq. (8) to write,

$$
\operatorname{Tr}_{A}^{i n c}=\frac{1}{A}\left|\frac{\mathcal{M}_{\gamma^{*} A \rightarrow V X}\left(s, Q^{2}\right)}{\mathcal{M}_{\gamma^{*} N \rightarrow V N}\left(s, Q^{2}\right)}\right|^{2}
$$

\subsection{The LC Green function approach}

One should decompose the physical photon $\left|\gamma^{*}\right\rangle$ into different Fock states, namely, the bare photon $\left|\gamma^{*}\right\rangle_{0},|\bar{q} q\rangle,|\bar{q} q G\rangle$, etc. The higher states containing gluons are vital to describe the energy dependence of the photoproduction reaction on a nucleon. As far as nuclear effects are concerned, those Fock components also lead to gluon shadowing. However, as we mentioned above, these fluctuations are heavier and have a shorter coherence time (lifetime) than the lowest $|\bar{q} q\rangle$ state. Therefore, at medium energies only $|\bar{q} q\rangle$ fluctuations of the photon matter. Gluon shadowing related to the higher Fock states will be considered later.

Propagation of an interacting $\bar{q} q$ pair in a nuclear medium is described by the Green function satisfying the evolution Eq. (19). However, the potential in this case acquires an imaginary part which represents absorption in the medium (see (11) for notations),

$$
\operatorname{Im} V_{\bar{q} q}\left(z_{2}, \vec{r}, \alpha\right)=-\frac{\sigma_{\bar{q} q}(\vec{r}, s)}{2} \rho_{A}\left(b, z_{2}\right) .
$$

The evolution equation (19) with the potential $V_{\bar{q} q}\left(z_{2}, \vec{r}_{2}, \alpha\right)$ containing this imaginary part was used in [54, 20], and nuclear shadowing in deep-inelastic scattering was calculated in good agreement with data.

The analytical solution of Eq. (21) is only known for the harmonic oscillator potential $V(r) \propto$ $r^{2}$. To keep the calculations reasonably simple we are forced to use the dipole approximation,

$$
\sigma_{\bar{q} q}(r, s)=C(s) r^{2}
$$

The energy dependent factor $C(s)$ is adjusted to reproduce correctly nuclear effects in the limit of very long CL $l_{c} \gg R_{A}$ (the so called "frozen" approximation), when

$$
G_{\bar{q} q}\left(z_{1}, \vec{r}_{1} ; z_{2}, \vec{r}_{2}\right) \Rightarrow \delta\left(\vec{r}_{1}-\vec{r}_{2}\right) \exp \left[-\frac{1}{2} \sigma_{\bar{q} q}\left(r_{1}\right) \int_{z_{1}}^{z_{2}} d z \rho_{A}(b, z)\right],
$$


where the dependence of the Green function on impact parameter is dropped. The details are described in Appendix A.

With the potential Eqs. (45) - (46) the solution of Eq. (19) has the same form as Eq. (21), except that one should replace $\omega \Rightarrow \Omega$, where

$$
\Omega=\frac{\sqrt{a^{4}(\alpha)-i \rho_{A}(b, z) \nu \alpha(1-\alpha) C(s)}}{\nu \alpha(1-\alpha)} .
$$

Guided by the uncertainty principle and the Lorentz transformation one can estimate the coherence time as in Eq. (3), where the effective mass of the $\bar{q} q$ pair is replaced by the vector meson mass. One can see the presence of a coherence length in the kinetic term of the evolution equation Eq. (19). Indeed, the effective mass squared of a $\bar{q} q$ pair is $M_{\bar{q} q}^{2}=\left(m_{q}^{2}+k_{T}^{2}\right) / \alpha(1-\alpha)$. This is what the kinetic term consists of when the transverse momentum squared of the quark is replaced by $k_{T}^{2} \Rightarrow \Delta_{r}$. This dynamically varying effective mass controls the CL defined by the Green function, as compared to the oversimplified Eq. (3) for the CL as given by the fixed mass $m_{V}$. One can explicitly see the static part $Q^{2}+m_{q}^{2} / \alpha(1-\alpha)$ of the coherence length in the last phase shift factor in the Green function in Eq. (21).

Depending on the value of $l_{c}$ one can distinguish different regimes:

(i) The CL is much shorter than the mean nucleon spacing in a nucleus $\left(l_{c} \rightarrow 0\right)$. In this case $G\left(z_{2}, \vec{r}_{2} ; z_{1}, \vec{r}_{1}\right) \rightarrow \delta\left(z_{2}-z_{1}\right)$ since strong oscillations suppress propagation of the $\bar{q} q$ over longer distances. In this case the formation time of the meson wave function is very short as well, since it is described by the same Green function and is controlled by the formation time as given in Eq. (2). Apparently, for light vector mesons $l_{f} \sim l_{c}$, so both must be short. In this case nuclear transparency is given by the simple formula Eq. (4) corresponding to the Glauber approximation?.

(ii) In the intermediate case $l_{c} \rightarrow 0$, but $l_{f} \sim R_{A}$, which can only be realized for heavy flavor quarkonia, the formation of the meson wave function is described by the Green function

\footnotetext{
${ }^{3}$ Note that the optical approximation is used throughout this paper only for the sake of easy reading. For numerical calculations we replace the exponential by a more realistic expression, $\exp \left(-\sigma T_{A}\right) \Rightarrow\left(1-\sigma T_{A} / A\right)^{A-1}$. This has also been done in all of our previous publications, contrary to what is stated in [55].
} 
and the numerator of the nuclear transparency ratio Eq. (44) has the form [8],

$$
\left|\mathcal{M}_{\gamma^{*} A \rightarrow V X}\left(s, Q^{2}\right)\right|_{l_{c} \rightarrow 0 ; l_{f} \sim R_{A}}^{2}=\int d^{2} b \int_{-\infty}^{\infty} d z \rho_{A}(b, z)\left|F_{1}(b, z)\right|^{2}
$$

where

$$
F_{1}(b, z)=\left.\int_{0}^{1} d \alpha \int d^{2} r_{1} d^{2} r_{2} \Psi_{V}^{*}\left(\vec{r}_{2}, \alpha\right) G\left(z^{\prime}, \vec{r}_{2} ; z, \vec{r}_{1}\right) \sigma_{\bar{q} q}\left(r_{1}, s\right) \Psi_{\gamma^{*}}\left(\vec{r}_{1}, \alpha\right)\right|_{z^{\prime} \rightarrow \infty}
$$

This expression is illustrated in Fig. 3a. The photon creates at the point $z$ a colorless $\bar{q} q$ pair

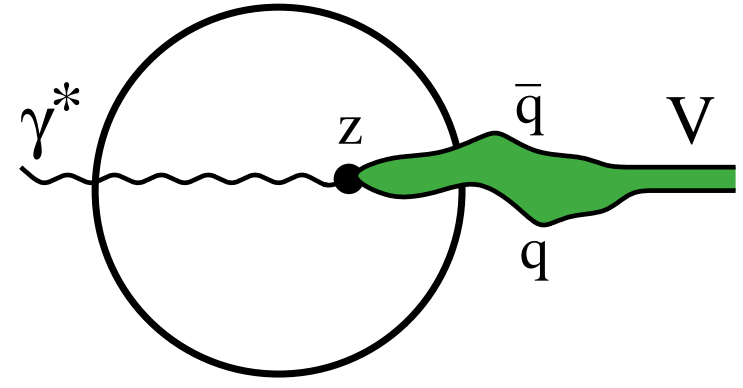

a

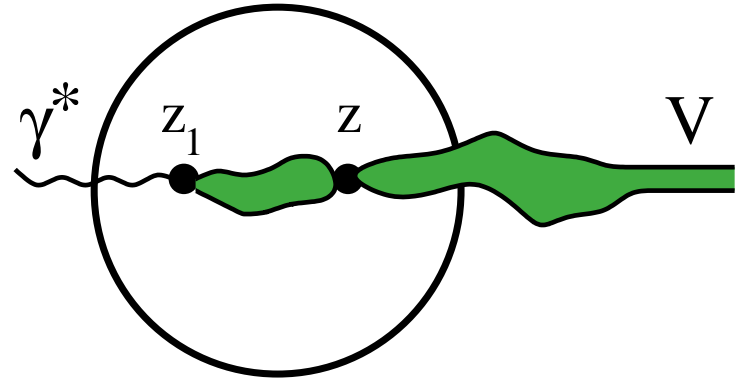

b

Figure 3: The incident virtual photon produces incoherently at the point $z$ (quasielastic scattering) the colorless $\bar{q} q$ pair which then evolves propagating through the nucleus and forms the $V$-meson wave function (a). Alternatively, the photon can first produce diffractively and coherently at the point $z_{1}$ the colorless $\bar{q} q$ which then experiences quasielastic scattering at the point $z$ (b). Propagation of the $\bar{q} q$ pair is described by the Green function (shaded areas).

with transverse separation $\vec{r}_{1}$. The quark and antiquark then propagate through the nucleus along different trajectories and end up with a separation $\vec{r}_{2}$. The contributions from different paths are summed up giving rise to the Green function $G\left(z^{\prime}, \vec{r}_{2} ; z, \vec{r}_{1}\right)$ which is convoluted in (49) with the wave functions of $\gamma^{*}$ and $V$. This is the path integral technique suggested in [8].

(iii) $l_{c} \gg R_{A}$ (in fact, it is more correct to compare with the mean free path of the $\bar{q} q$ in a nuclear medium if the latter is shorter than the nuclear radius). In this case $G\left(z_{2}, \vec{r}_{2} ; z_{1}, \vec{r}_{1}\right) \rightarrow$ 
$\delta\left(\vec{r}_{2}-\vec{r}_{1}\right)$, i.e. all fluctuations of the transverse $\bar{q} q$ separation are "frozen" by Lorentz time dilation. Then, the numerator on the r.h.s. of Eq. (44) takes the form [8],

$$
\begin{aligned}
\left|\mathcal{M}_{\gamma^{*} A \rightarrow V X}\left(s, Q^{2}\right)\right|_{l_{c} \gg R_{A}}^{2} & =\int d^{2} b T_{A}(b) \mid \int d^{2} r \int_{0}^{1} d \alpha \\
& \times\left.\Psi_{V}^{*}(\vec{r}, \alpha) \sigma_{\bar{q} q}(r, s) \exp \left[-\frac{1}{2} \sigma_{\bar{q} q}(r, s) T_{A}(b)\right] \Psi_{\gamma^{*}}\left(\vec{r}, \alpha, Q^{2}\right)\right|^{2}
\end{aligned}
$$

In this case the $\bar{q} q$ attenuates with a constant absorption cross section like in the Glauber model, except that the whole exponential is averaged rather than just the cross section in the exponent. The difference between the results of the two prescriptions are the well known inelastic corrections of Gribov [2].

(iv) The main and new results of the present paper address the general case with no restrictions for either $l_{c}$ or $l_{f}$. No theoretical tool has been developed so far beyond the limits (i) - (iii) discussed above neither of which can be applied to electroproduction of light vector mesons at the medium high energies of HERMES and JLab.

Even within the VDM the Glauber model expression interpolating between the limiting cases of low [(i), (ii)] and high [(iii)] energies has been derived only recently [16]. We generalize that formalism to the LC dipole approach, and the incoherent photoproduction amplitude is represented as a sum of two terms [56] illustrated in Fig. 3,

$$
\left|\mathcal{M}_{\gamma^{*} A \rightarrow V X}\left(s, Q^{2}\right)\right|^{2}=\int d^{2} b \int_{-\infty}^{\infty} d z \rho_{A}(b, z)\left|F_{1}(b, z)-F_{2}(b, z)\right|^{2} .
$$

The first term $F_{1}(b, z)$ introduced above in Eq. (50) is represented by Fig. 3a. Alone it would correspond to the short $l_{c}$ limit (ii). The second term $F_{2}(b, z)$ in (52) corresponds to the situation illustrated in Fig. 3b. The incident photon produces a $\bar{q} q$ pair diffractively and coherently at the point $z_{1}$ prior to incoherent quasielastic scattering at point $z$. The LC Green functions describe the evolution of the $\bar{q} q$ over the distance from $z_{1}$ to $z$ and further on, up to the formation of the meson wave function. Correspondingly, this term has the form,

$$
\begin{aligned}
F_{2}(b, z) & =\frac{1}{2} \int_{-\infty}^{z} d z_{1} \rho_{A}\left(b, z_{1}\right) \int_{0}^{1} d \alpha \int d^{2} r_{1} d^{2} r_{2} d^{2} r \Psi_{V}^{*}\left(\vec{r}_{2}, \alpha\right) \\
& \times G\left(z^{\prime} \rightarrow \infty, \vec{r}_{2} ; z, \vec{r}\right) \sigma_{\bar{q} q}(\vec{r}, s) G\left(z, \vec{r} ; z_{1}, \vec{r}_{1}\right) \sigma_{\bar{q} q}\left(\vec{r}_{1}, s\right) \Psi_{\gamma^{*}}\left(\vec{r}_{1}, \alpha\right) .
\end{aligned}
$$


Eq. (52) correctly reproduces the limits (i) - (iii). Indeed, at $l_{c} \rightarrow 0$ the second term $F_{2}(b, z)$ vanishes because of strong oscillations, and Eq. (52) reproduces the Glauber expression Eq. (4). On the other hand, at $l_{c} \gg R_{A}$ the phase shift in the Green functions can be neglected and they acquire the simple form $G\left(z_{2}, \vec{r}_{2} ; z_{1}, \vec{r}_{1}\right) \rightarrow \delta\left(\vec{r}_{2}-\vec{r}_{1}\right)$. In this case the integration over longitudinal coordinates in Eqs. (50) and (53) can be performed explicitly and the asymptotic expression Eq. (51) is recovered as well. Moreover, if one uses a constant dipole cross section $\sigma_{\bar{q} q}(\rho)=\sigma_{\text {tot }}^{V N}$, then Eq. (52) recovers the general Glauber expression derived in [16].

\subsection{Data for incoherent production: CT or coherence?}

Exclusive incoherent electroproduction of vector mesons off nuclei has been suggested in [57 as a sensitive way to detect $\mathrm{CT}$. Increasing the photon virtuality $Q^{2}$ one squeezes the produced $\bar{q} q$ wave packet. Such a small colorless system propagates through the nucleus with little attenuation, provided that the energy is sufficiently high $\left(l_{f} \gg R_{A}\right)$ the fluctuations of the $\bar{q} q$ separation are frozen by Lorentz time dilation. Thus, a rise of nuclear transparency $\operatorname{Tr}_{A}^{i n c}\left(Q^{2}\right)$ with $Q^{2}$ should signal CTP. Indeed, such a rise was observed in the E665 experiment at Fermilab for exclusive production of $\rho^{0}$ mesons off nuclei by a muon beam. This has been claimed in 61] to be a manifestation of CT.

However, one should be cautious to avoid mixing up the expected signal for CT with the effect of coherence length [63, 16]. Indeed, if the coherence length varies from long to short compared to the nuclear size the nuclear transparency rises because the length of the path in

\footnotetext{
${ }^{4}$ Note that Eq. (52) and its Glauber model analog in 16 include all coherent multiple scattering terms, contrary to a statement made in [55].

${ }^{5}$ This process has a definite advantage compared to quasielastic electron scattering (e,e'p) suggested in [58, 59] as a probe for CT. Indeed, in the latter case the energy of the photon correlates with its virtuality, $\nu \approx 2 m_{N} Q^{2}$, and one has to increase $Q^{2}$ just in order to increase $\nu$ and keep the size of the ejectile "frozen". This leads to a substantially diminished cross section, which is why no CT signal has been detected in this reaction so far (it is still possible to observe $\mathrm{CT}$ in this reaction at low energy studying the asymmetry of the quasielastic peak as function of $x_{B j}\left[60\right.$ ). In contrast, no correlation between $\nu$ and $Q^{2}$ exists in exclusive electroproduction of vector mesons.
} 
nuclear matter becomes shorter and the vector meson (or $\bar{q} q$ ) attenuates less. This happens when $Q^{2}$ increases at fixed $\nu$. One should carefully disentangle these two phenomena.

Long CL. It has been checked in [63] that the coherence length at the kinematics of the E665 experiment is sufficiently long to neglect its variation with $Q^{2}$ and to use the "frozen" approximation, except at the highest values of $Q^{2} \gtrsim 5 \mathrm{GeV}^{2}$. We calculated nuclear transparency, $\operatorname{Tr}_{A}^{i n c}$, of incoherent (quasielastic) $\rho^{0}$ production using Eq. (52) and the simplified "frozen" approximation Eqs. (47) - (51). The results are depicted in Fig. 4 by solid and dashed curves respectively. One can see that fluctuations of the size of the $\bar{q} q$ pair become important only

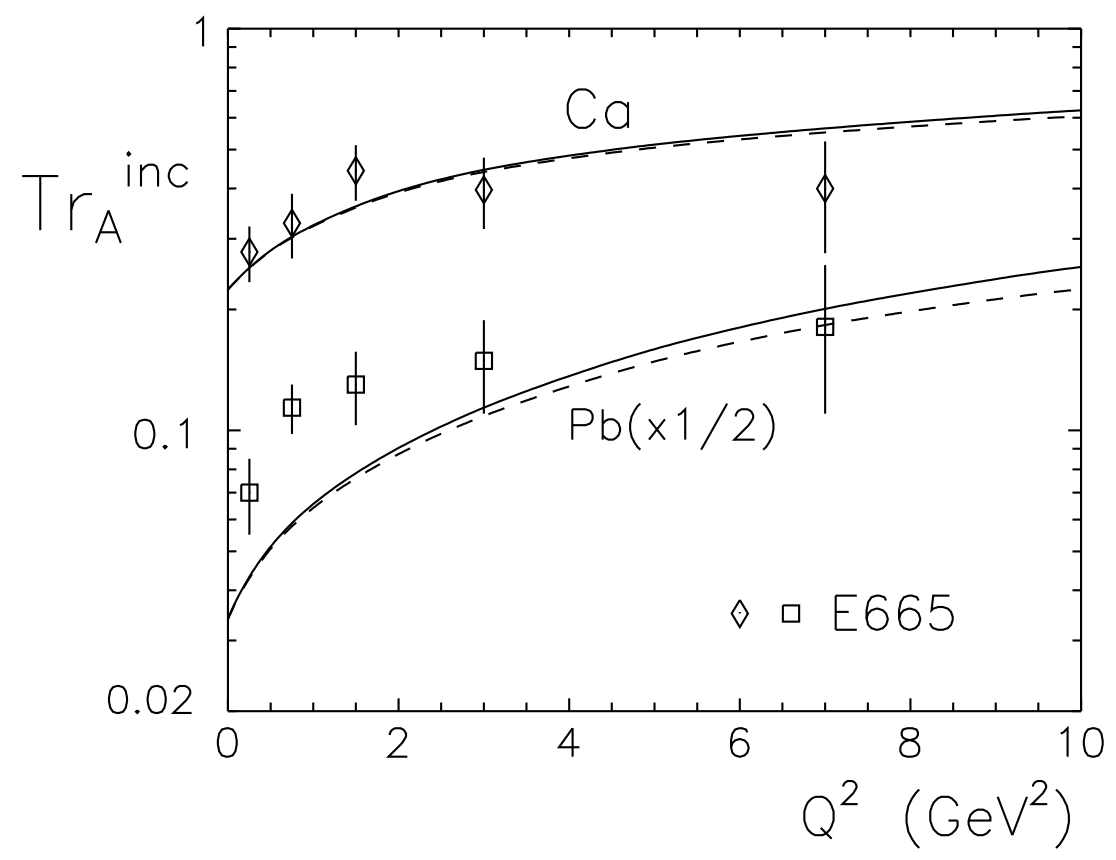

Figure 4: $Q^{2}$ - dependence of nuclear transparency for lead and calcium $\operatorname{Tr}_{P b}$ and $\operatorname{Tr}_{C a}$ vs. The experimental points are from the E665 experiment [61]. Both the curves and data for lead are rescaled by the factor 1/2. Solid and dashed curves show our results using the LC Green function approach Eq. (52) and the "frozen" approximation Eq. (51) respectively.

at high $Q^{2}$ causing a separation of the solid and dashed curves. At smaller $Q^{2}$ the observed variation of $\operatorname{Tr}_{A}^{i n c}\left(Q^{2}\right)$ is a net manifestation of CT. The agreement with our model is surpris- 
ingly good for calcium, while we underestimate the nuclear transparency at small $Q^{2}$ for lead. This may be a manifestation of large Coulomb corrections as found in [62], which are of the order $\alpha_{e m} Z \approx 0.6$ for lead. These corrections lead to a considerable deviation from the Born, one-photon approximation employed in [61] in order to obtain data for $\gamma^{*} A \rightarrow \rho^{0} X$ (depicted in Fig. (4) from raw data for $\mu A \rightarrow \mu^{\prime} \rho^{0} X$. This important problem needs further study.

Medium long CL. The same process of incoherent electroproduction of $\rho^{0}$ is under study at lower energies, in the HERMES experiment at HERA and at JLab. In this case one should carefully discriminate between the effects of CT and CL [63, 16]. A simple prescription [9] to eliminate the effect of CL from the data on the $Q^{2}$ dependence of nuclear transparency is to bin the data in a way which keeps $l_{c}=$ const. It means that one should vary simultaneously $\nu$ and $Q^{2}$ maintaining the CL Eq. (3) constant,

$$
\nu=\frac{1}{2} l_{c}\left(Q^{2}+m_{V}^{2}\right) .
$$

In this case the Glauber model predicts a $Q^{2}$ independent nuclear transparency, and any rise with $Q^{2}$ would signal CT [9].

The LC Green function technique incorporates both the effects of coherence and formation. We performed calculations of $\operatorname{Tr}_{A}^{i n c}\left(Q^{2}\right)$ at fixed $l_{c}$ starting from different minimal values of $\nu$, which correspond to real photoproduction in Eq. (54),

$$
\nu_{\min }=\frac{1}{2} l_{c} m_{V}^{2}
$$

The results for incoherent production of $\rho$ and $\phi$ at $\nu_{\min }=0.9,2,5$ and $10 \mathrm{GeV}\left(l_{c}=\right.$ 0.6-6.75 fm) are presented in Fig. 5 for nitrogen, krypton and lead. We use the nonperturbative $\mathrm{LC}$ wave function of the photon with the parameters of the LC potential $a_{0,1}$ fixed in accordance with Eq. (31) at $v=1 / 2$. The $u$ and $d$ quarks are assumed to be massless, but we use $m_{s}=0.15 \mathrm{GeV}$. Nuclear transparency for $\phi$ is stronger than for $\rho$ as one could expect, but the difference is not significant. In what follows we discuss only our results for $\rho$.

For $\rho$-mesons the predicted variation of nuclear transparency with $Q^{2}$ at fixed $l_{c}$ is much stronger than was found in [9]. Those calculations have been done in the hadronic representation which is quite challenging due to the necessity to know all the diagonal and off diagonal 


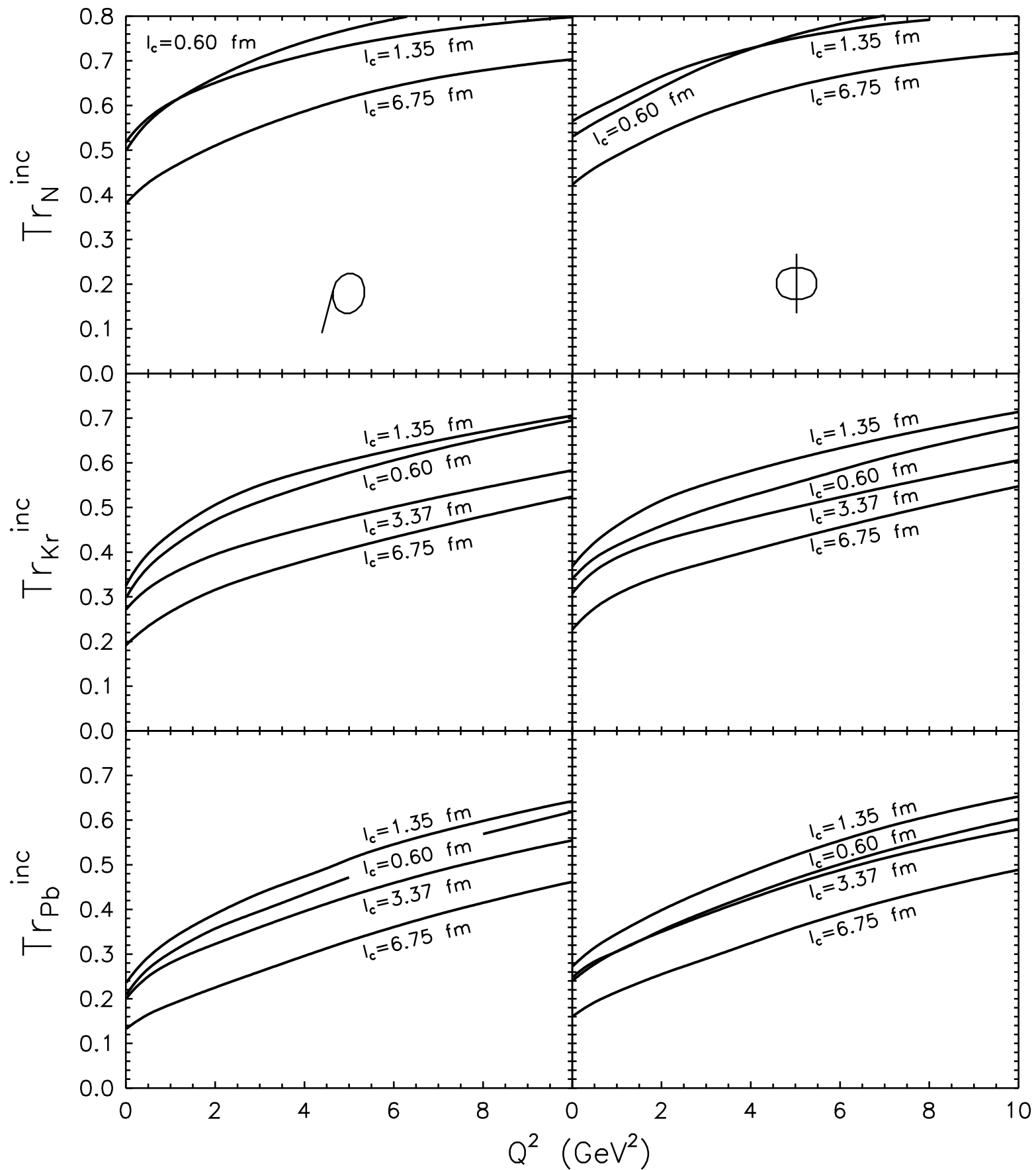

Figure 5: $Q^{2}$ dependence of the nuclear transparency $\operatorname{Tr}_{A}^{i n c}$ for exclusive electroproduction of $\rho$ (left) and $\phi$ (right) mesons on nuclear targets ${ }^{14} N$, ${ }^{84} \mathrm{Kr}$ and ${ }^{207} \mathrm{~Pb}$ (from top to bottom). The $C L$ is fixed at $l_{c}=0.60,1.35$, 3.37 and $6.75 \mathrm{fm}$. 
diffractive amplitudes for the vector meson and its excitations, as well as all photoproduction amplitudes. The predictions made in [9] were based on the two-coupled-channel model without any estimate of the accuracy of such an approximation. According to quark-hadron duality the LC Green function method is equivalent to the exact solution of the general multi-channel problem in the hadronic representation. The comparison therefore demonstrates that the twochannel approximation substantially underestimates the effect of color transparency.

To see the scale of the theoretical uncertainty of our model [23 for nonperturbative effects we compare in Fig. 6 the results for the $\rho$-meson obtained using the nonperturbative (solid curves) and perturbative photon wave functions Eq. (14) with $m_{q}=0.15 \mathrm{GeV}$ (dashed curves). The difference between the two sets of curves is insignificant.

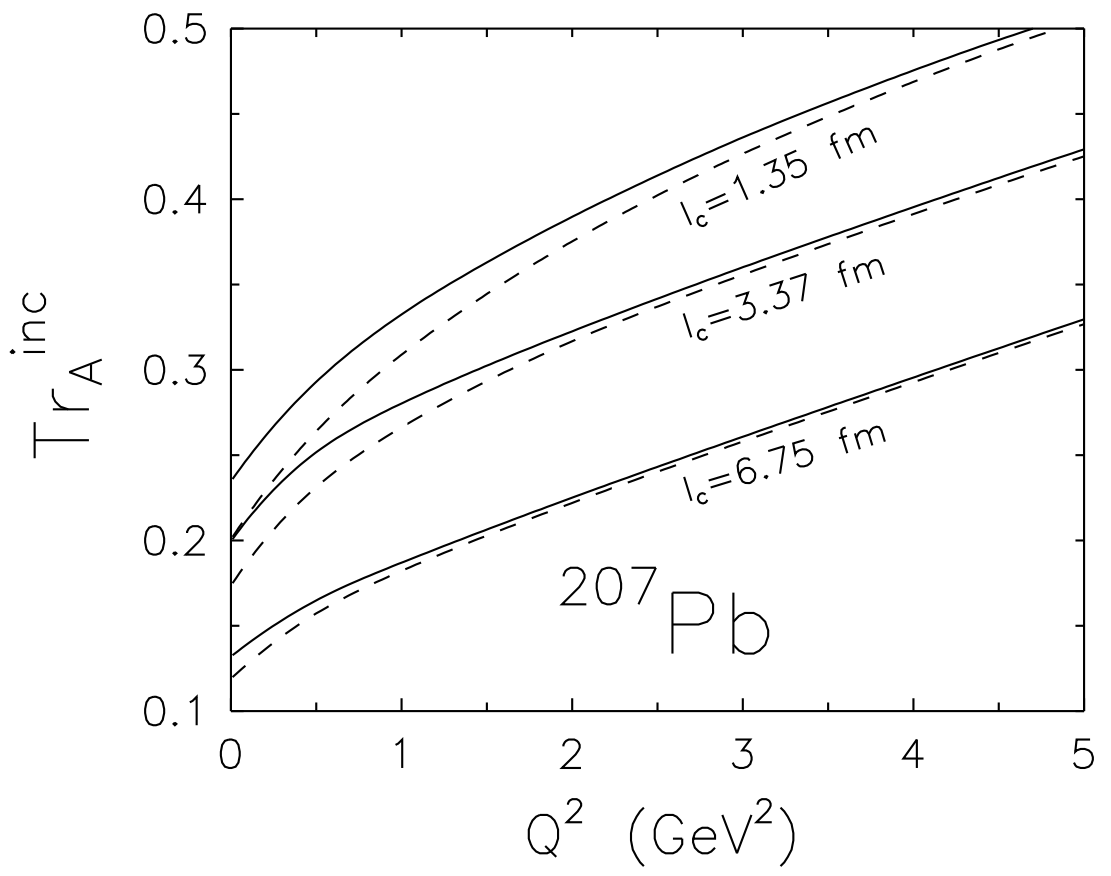

Figure 6: The same as in Fig. 5 for lead, but calculated with both nonperturbative (solid curves) and perturbative (dashed) wave functions of the photon.

Motivated by the too weak signal predicted for CT it was suggested in [9] that instead one can study the effect of coherence which has never been observed experimentally. Indeed, it was 
found in [64] that data for nuclear transparency for $\rho$ production plotted as function of $l_{c}$ well agree with what was predicted in [16] to be the effect of the CL. Now we find a rather strong signal of CT which may also affect the $l_{c}$ dependence of $\operatorname{Tr}_{A}^{i n c}$ and cause a deviation from the Glauber model expectations. We therefore revise the previous conclusions [9, 64].

In the VDM-Glauber model nuclear transparency is a function of $l_{c}$ only (neglecting the weak energy dependence of $\left.\sigma_{t o t}^{V N}\right)$, however it becomes a function of two variables, $\operatorname{Tr}_{A}^{i n c}\left(l_{c}, Q^{2}\right)$, as soon as CT effects are involved. Therefore, our current predictions for the $l_{c}$ dependence of $\operatorname{Tr}_{A}^{i n c}$ vary with $Q^{2}$. They are plotted by dashed curves in Fig. 0 for different fixed values of $Q^{2}=0.5,1,2,3,5 \mathrm{GeV}^{2}$ (from bottom to top) for nitrogen and krypton (left and right boxes, respectively). The nonperturbative wave function of the photon was used as for Fig.

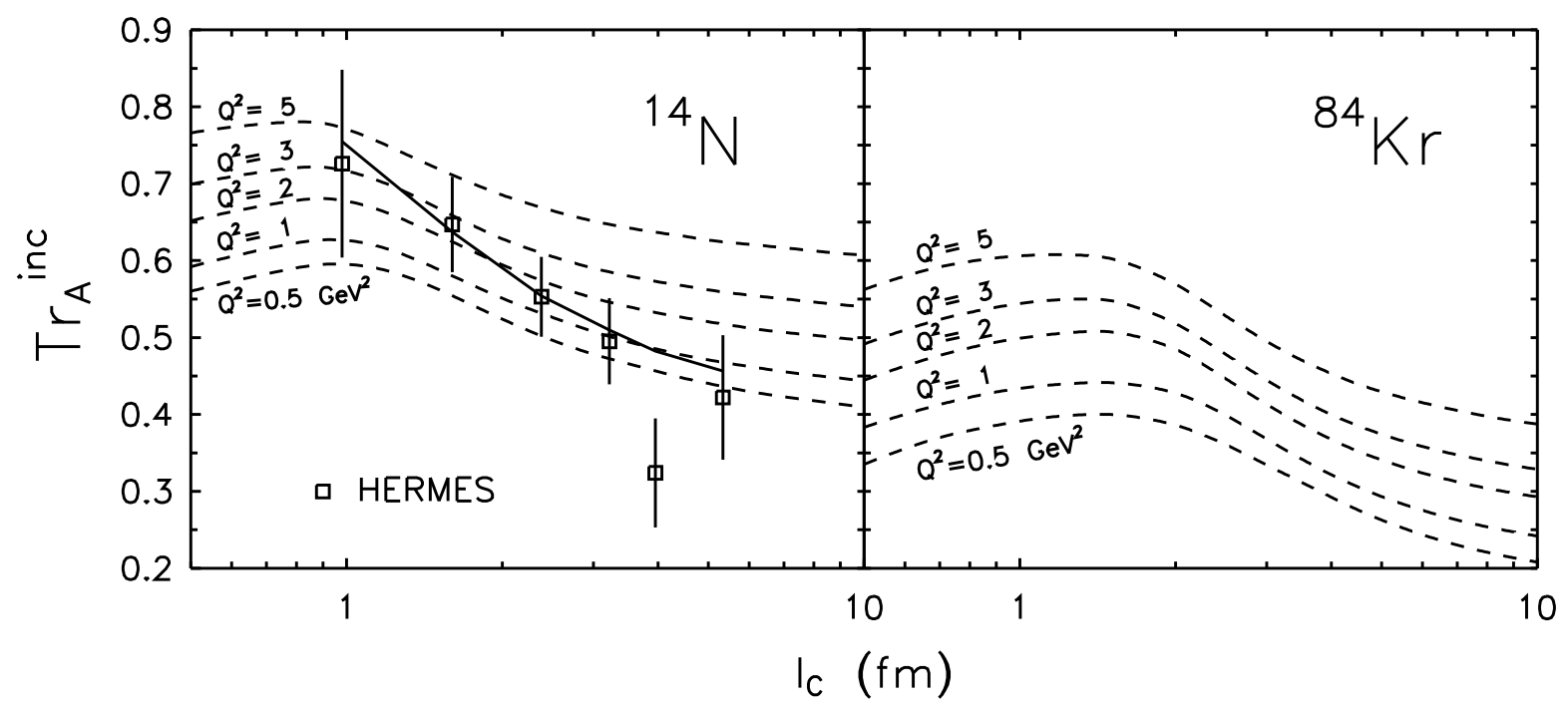

Figure 7: Nuclear transparency for incoherent electroproduction of $\rho$ off nuclei, nitrogen and krypton, as function of $l_{c}$ at fixed $Q^{2}=$ $0.5,1,2,3,5 \mathrm{GeV}^{2}$. The solid curve is calculated at the mean values of $l_{c}$ and $Q^{2}$ corresponding to each experimental point 64, 65.

We do not show the results obtained with the perturbative wave function since they are pretty much the same, except in the region of small $Q^{2}$ and short $l_{c}$ where they are about $10 \%$ lower 
that the nonperturbative results.

The experimental points for nitrogen [64] which are plotted in Fig. [ ] correspond to different mean experimental values of $Q^{2}$ [65]. This $Q^{2}-l_{c}$ correlation is incorporated in our calculations, and the results depicted by the solid curve agree well with the data.

We thus arrive at the conclusion that the two quite different approaches, the VDM based Glauber model and QCD based LC Green function formalism, both provide good agreement with the HERMES data. This could not be possible if the data were plotted as function of $l_{c}$ at fixed $Q^{2}$. The observed agreement with the Glauber model seems to be accidental and a result of the $Q^{2}-l_{c}$ correlation in the data.

In order to discriminate between the two approaches one should plot the data differently. Fig. 0 gives hope that the data are sufficiently accurate to detect a signal of CT if they are properly analyzed. Also additional data for krypton should soon become available from HERMES.

The expected signal for CT is a nonzero derivative $d \ln \left[\operatorname{Tr}_{A}\left(Q^{2}\right)\right] / d Q^{2}$ which is predicted in Fig. 5 to be similar for different nuclei and different values of $l_{c}$. One can make use of this fact and perform a common fit to all available data with only one parameter which is the slope of the $Q^{2}$ dependence of nuclear transparency. The value of the logarithmic slope for the mid values, $Q^{2} \approx 1-2 \mathrm{GeV}^{2}$, of the HERMES kinematical range for $\rho$ production is expected to vary within the interval

$$
\left.\frac{1}{\operatorname{Tr}_{A}^{i n c}\left(Q^{2}\right)} \frac{d \operatorname{Tr}_{A}^{i n c}\left(Q^{2}\right)}{d Q^{2}}\right|_{l_{c}=\text { const }} \approx \begin{aligned}
& 0.07-0.11 \mathrm{GeV}^{-2} \text { for }{ }^{14} N \\
& 0.14-0.17 \mathrm{GeV}^{-2} \text { for }{ }^{84} \mathrm{Kr}
\end{aligned}
$$

for $l_{c}=0.60-6.75 \mathrm{fm}$. Similar, but somewhat smaller values of the logarithmic $Q^{2}$-slope are expected for $\phi$.

The curves in Fig. 5 demonstrate an interesting property. The slope of the $Q^{2}$ dependence is steeper at small $Q^{2}$ and $l_{c}$. For instance, the logarithmic derivative Eq. (56) equals 0.09 at $l_{c}=$ $0.6 \mathrm{fm}$, but is smaller, 0.07 at $l_{c}=1.35 \mathrm{fm}$ This fact might be in variance with naive intuitive expectations. Indeed, $l_{c}=0.6 \mathrm{fm}$ is short compared to th mean spacing of the bound nucleons. Since $l_{f} \sim l_{c}$ at low $Q^{2}$ one might expect the Glauber model to be a good approximation in this 


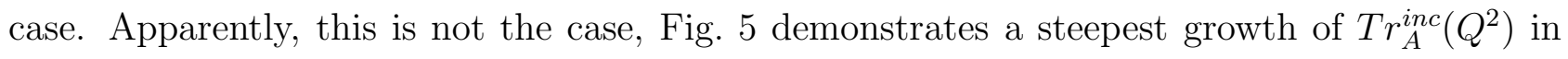
this region. One can understand this as follows. If $l_{c}$ is long, like in Fig. 团, then the formation length is long too, $l_{f} \gtrsim l_{c} \gg R_{A}$, and nuclear transparency rises with $Q^{2}$ only because the mean transverse separation of the $\bar{q} q$ fluctuations decreases. If, however, $l_{c} \lesssim R_{A}$ and fixed, the photon energy rises with $Q^{2}$ according to Eq. (54) and the formation length Eq. (22) rises as well. Thus, these two effects, the $Q^{2}$ dependence of $l_{f}$ and the $\bar{q} q$ transverse size, add up and lead to a steeper growth of $\operatorname{Tr}_{A}^{i n c}\left(Q^{2}\right)$ for short $l_{c}$.

One should conclude from this consideration that the CT effects are more pronounced at low than at high energies. This observation adds to the motivation for experimental searches for CT at HERMES and JLab.

We also calculated the energy dependence of nuclear transparency at fixed $Q^{2}$. The results for nitrogen and lead are shown by dashed curves in Fig. 8 for different values of $Q^{2}$. The

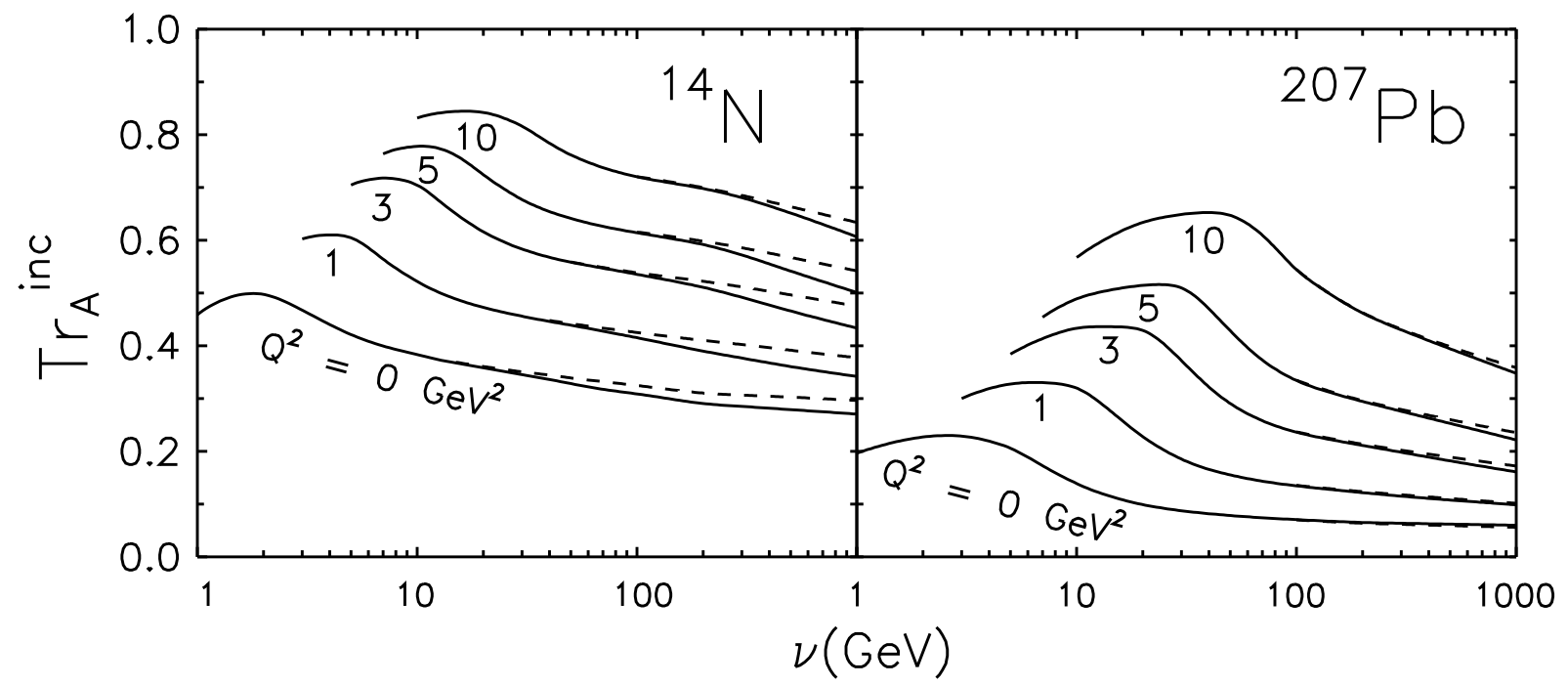

Figure 8: Nuclear transparency for incoherent electroproduction $\gamma^{*} A \rightarrow$ $\rho^{0} A$ as function of energy at $Q^{2}=0,1,3,5,10 \mathrm{GeV}^{2}$ for nitrogen and lead. The solid and dashed curves correspond to calculations with and without gluons shadowing, respectively.

interesting feature is the presence of a maximum of transparency at some energy. It results 
from the interplay of coherence and formation effects. Indeed, the FL rises with energy leading to an increasing nuclear transparency. At some energy, however, the effect of CL which is shorter than the FL, is switched on leading to a growth of the path length of the $\bar{q} q$ in the nucleus, i.e. to a suppression of transparency. The maxima in the $l_{c}$ dependence of nuclear transparency depicted in Fig. 7 are of the same nature. This also explains the unusual ordering of curves calculated for different values of $l_{c}$ as is depicted in Fig. 5 .

\section{Coherent production of vector mesons}

\subsection{The formalism}

If electroproduction of a vector meson leaves the target intact the process is usually called coherent or elastic. The mesons produced at different longitudinal coordinates and impact parameters add up coherently. This fact considerably simplifies the expressions for the cross sections compared to the case of incoherent production. The integrated cross section has the form,

$$
\sigma_{A}^{c o h} \equiv \sigma_{\gamma^{*} A \rightarrow V A}^{c o h}=\int d^{2} q\left|\int d^{2} b e^{i \vec{q} \cdot \vec{b}} \mathcal{M}_{\gamma^{*} A \rightarrow V A}^{c o h}(b)\right|^{2}=\int d^{2} b\left|\mathcal{M}_{\gamma^{*} A \rightarrow V A}^{c o h}(b)\right|^{2}
$$

where

$$
\mathcal{M}_{\gamma^{*} A \rightarrow V A}^{c o h}(b)=\int_{-\infty}^{\infty} d z \rho_{A}(b, z) F_{1}(b, z),
$$

with the function $F_{1}(b, z)$ defined in (50).

One should not use Eq. (44) for nuclear transparency any more since the $t$-slopes of the differential cross sections for nucleon and nuclear targets are different and do not cancel in the ratio. Therefore, the nuclear transparency also includes the slope parameter $B_{\gamma^{*} N}$ for the process $\gamma^{*} N \rightarrow V N$

$$
\operatorname{Tr}_{A}^{c o h}=\frac{\sigma_{A}^{c o h}}{A \sigma_{N}}=\frac{16 \pi B_{\gamma^{*} N} \sigma_{A}^{c o h}}{A\left|\mathcal{M}_{\gamma^{*} N \rightarrow V N}\left(s, Q^{2}\right)\right|^{2}}
$$

One can also define a $t$-dependent transparency for coherent electroproduction of vector 
mesons,

$$
\operatorname{Tr}_{A}^{c o h}(t)=\frac{d \sigma_{A}^{c o h} / d t}{A^{2} d \sigma_{N} /\left.d t\right|_{t=0}}
$$

where the differential cross section for coherent production $\gamma^{*} A \rightarrow V A$ reads

$$
\frac{d \sigma_{A}^{c o h}}{d t}=\frac{1}{16 \pi}\left|\int d^{2} b e^{i \vec{b} \cdot \vec{q}} \int_{-\infty}^{\infty} d z \rho_{A}(b, z) F_{1}(b, z)\right|^{2}
$$

with $F_{1}(b, z)$ defined in (50). This expression is simplified in the limit of long coherence time $\left(t=-q^{2}\right)$,

$$
\begin{aligned}
\left.\frac{d \sigma_{A}^{c o h}}{d t}\right|_{l_{c} \gg R_{A}} & =\frac{1}{4 \pi} \mid \int d^{2} b e^{i \vec{b} \cdot \vec{q}} \int d^{2} r\left\{1-\exp \left[-\frac{1}{2} \sigma_{\bar{q} q}(\vec{r}, s) T(b)\right]\right\} \\
& \times\left.\int_{0}^{1} d \alpha \Psi_{V}^{*}(\vec{r}, \alpha) \Psi_{\gamma^{*}}(\vec{r}, \alpha)\right|^{2},
\end{aligned}
$$

a form which resembles its VDM analogue [10].

\subsection{Comparison with data and predictions for coherent production}

Using Eq. (59) we can also calculate the normalized ratio of coherent cross sections on two nuclei $R_{c o h}\left(A_{1} / A_{2}\right)=\operatorname{Tr}_{A_{1}}^{c o h} / \operatorname{Tr}_{A_{2}}^{c o h}$. The results of calculations for $R_{c o h}(P b / C)$ and $R_{c o h}(C a / C)$ are depicted by solid curves in Fig. 9 as well as corresponding data from the E665 experiment 61] shown by squares and triangles, respectively. We performed calculations of $\operatorname{Tr}_{A}^{c o h}$ at mean photon energy $\bar{\nu}=138 \mathrm{GeV}$ with the $Q^{2}$ dependent slope given by Eq. (41). All effects of CL

and CT are included via the LC Green function formalism. For such a high energy one can think that the "frozen" approximation $l_{c} \gg R_{A}$ is good. In order to check how variation of the CL affects the nuclear transparency we repeated our calculations in the "frozen" approximation and plotted the results as dashed curves in Fig. 9. We see that the accuracy of this approximation is rather good for calcium, while for lead it significantly deviates from the exact result at $Q^{2} \gtrsim 2 \mathrm{GeV}^{2}$. The reason is obvious, the heavier the nucleus, the less the approximation $l_{c} \gg R_{A}$ is fulfilled. We also see that the contraction of the CL with $Q^{2}$ causes an effect opposite to CT, namely nuclear transparency is suppressed rather than enhanced. Therefore, 


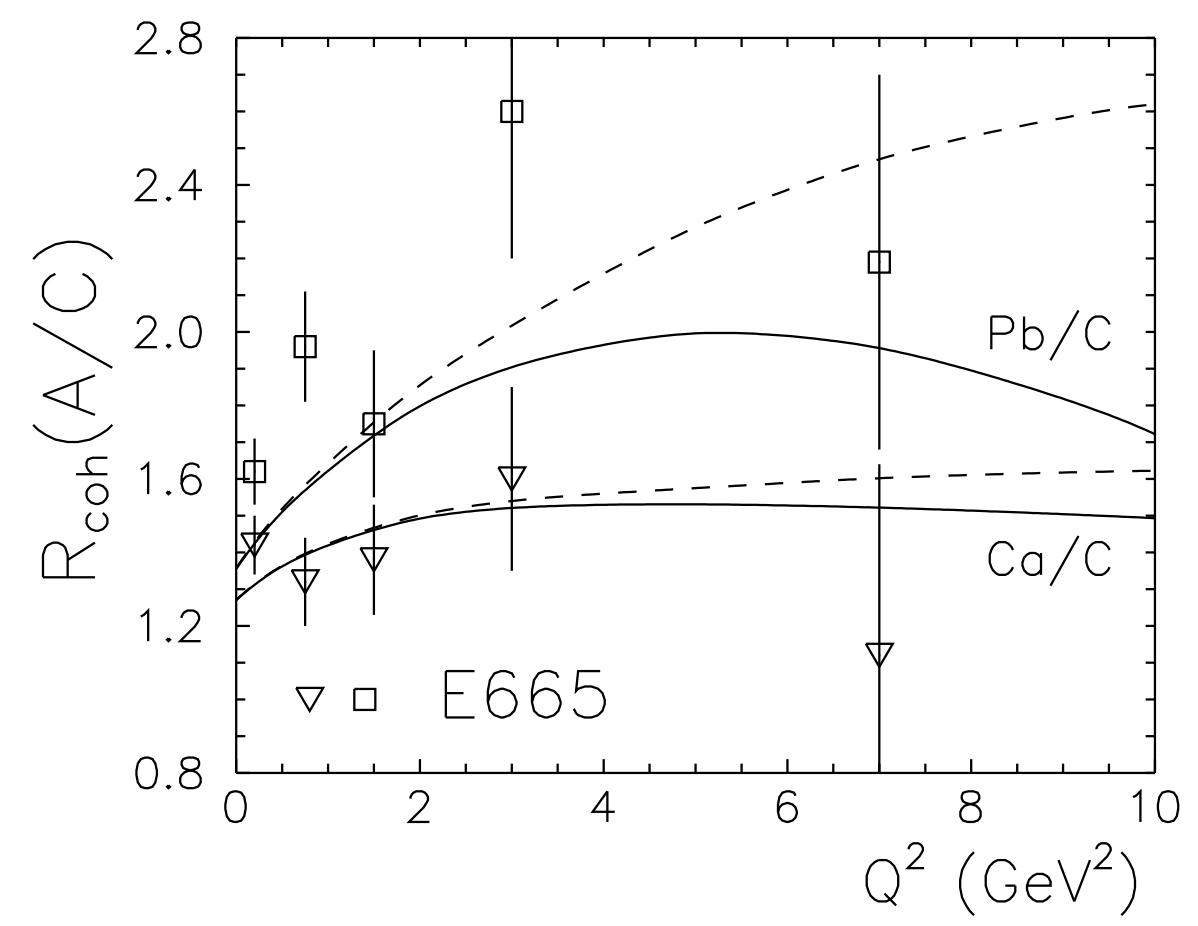

Figure 9: $Q^{2}$ - dependence of the total cross section ratio $R_{\text {coh }}(A / C)=$ $12 \sigma_{A}^{c o h} / A \sigma_{C}^{c o h}$ for the coherent process $\gamma^{*} A \rightarrow \rho^{0} A$. Experimental points are from E665 [61] for $\mathrm{Pb} / \mathrm{C}$ (squares) and $\mathrm{Ca} / \mathrm{C}$ (triangles). Solid curves include the variation of $l_{c}$ and $l_{f}$ with $Q^{2}$. Dashed curves are calculated in the "frozen" approximation $l_{c} \gg R_{A}$.

there is no danger that CL effects can mock CT, and one may think that this is an advantage of coherent compared to incoherent production [63]. However, at medium energy the suppression of nuclear transparency at short CL is so strong that no rise of nuclear transparency with $Q^{2}$ might be observable.

Note that in contrast to incoherent production where nuclear transparency is expected to saturate as $\operatorname{Tr}_{A}^{i n c}\left(Q^{2}\right) \rightarrow 1$ at large $Q^{2}$, for the coherent process nuclear transparency reaches a higher limit, $\operatorname{Tr}_{A}^{c o h}\left(Q^{2}\right) \rightarrow A^{1 / 3}$ (of course, $A^{1 / 3}$ is valid only for very large nuclei, otherwise it is an approximate number). The dashed curves in Fig. 9 nearly reach this upper limit at $Q^{2} \sim 10 \mathrm{GeV}^{2}$.

One can eliminate the effects of CL and single out the net CT effect in a way similar to 
what was suggested for incoherent reactions by selecting experimental events with $l_{c}=$ const. We calculated nuclear transparency for the coherent reaction $\gamma^{*} A \rightarrow \rho(\phi) A$ at fixed values of $l_{c}$. The results for $l_{c}=1.35,3.37,13.50 \mathrm{fm}$ are depicted in Fig. 10 for several nuclei. The effect

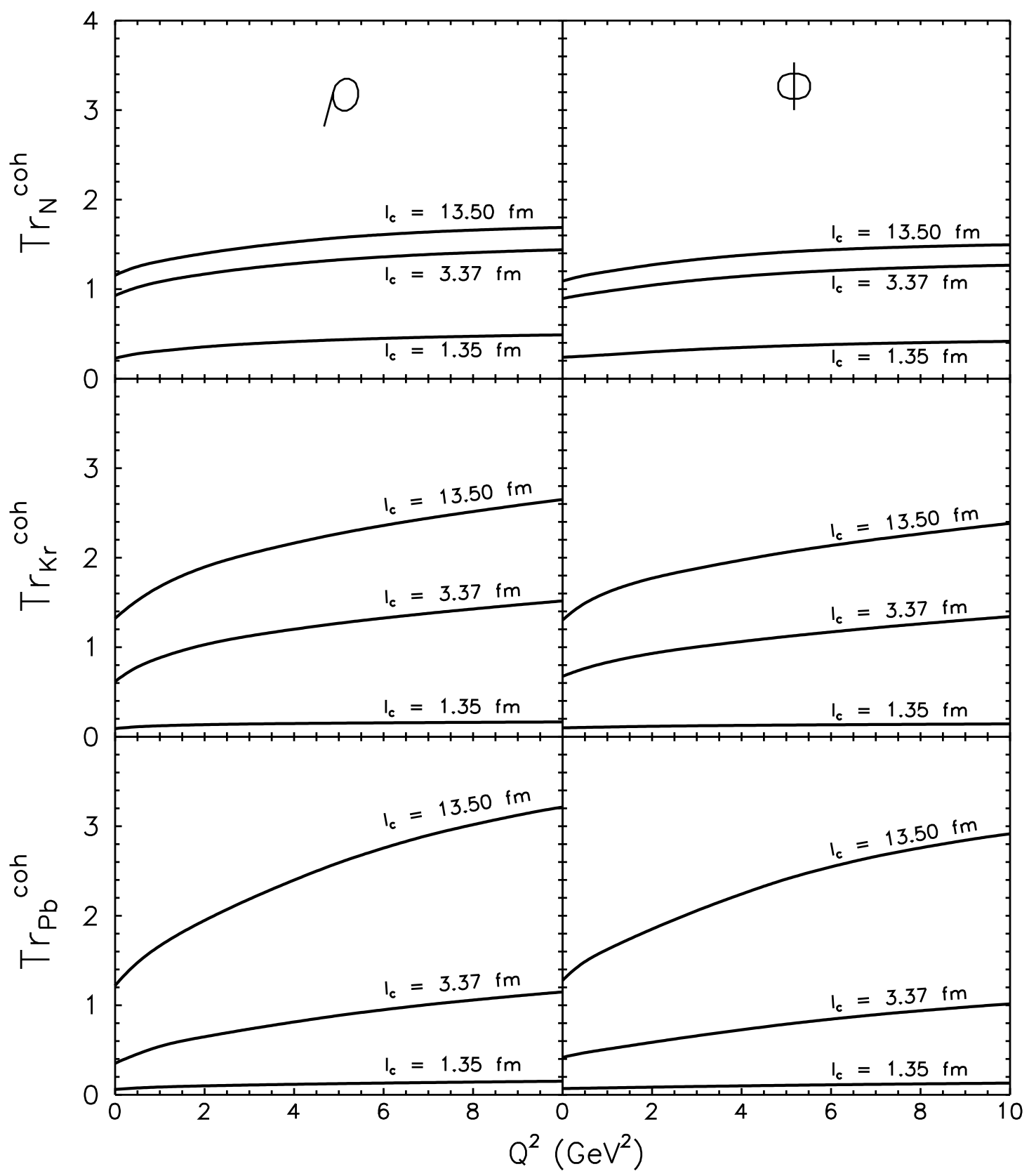

Figure 10: The same as in Fig. 5, but for coherent production of $\rho$ and $\phi$, $\gamma^{*} A \rightarrow V A$. 
is sufficiently large to be observable, the logarithmic derivative varies within the interval,

$$
\left.\frac{1}{\operatorname{Tr}_{A}^{c h h}\left(Q^{2}\right)} \frac{d \operatorname{Tr}_{A}^{c o h}\left(Q^{2}\right)}{d Q^{2}}\right|_{l_{c}=\text { const }} \approx \begin{aligned}
& 0.14-0.07 \mathrm{GeV}^{-2} \text { for }{ }^{14} N \\
& 0.10-0.15 \mathrm{GeV}^{-2} \text { for }{ }^{84} \mathrm{Kr}
\end{aligned}
$$

for $l_{c}=1.35-13.5 \mathrm{fm}$. Again, like in the case of incoherent production, the logarithmic derivative decreases at large $l_{c}$. The magnitude of the expected CT effect is similar to the value predicted for the incoherent production in Eq. (56) and is slightly smaller for phi than for $\rho$.

We also calculated nuclear transparency as function of energy at fixed $Q^{2}$. The results for $\rho$ produced coherently off nitrogen and lead are depicted by dashed curves in Fig. 11 at $Q^{2}=0,3,10 \mathrm{GeV}^{2} . \operatorname{Tr}_{A}^{c o h}$ is very small at low energy, what of course does not mean that

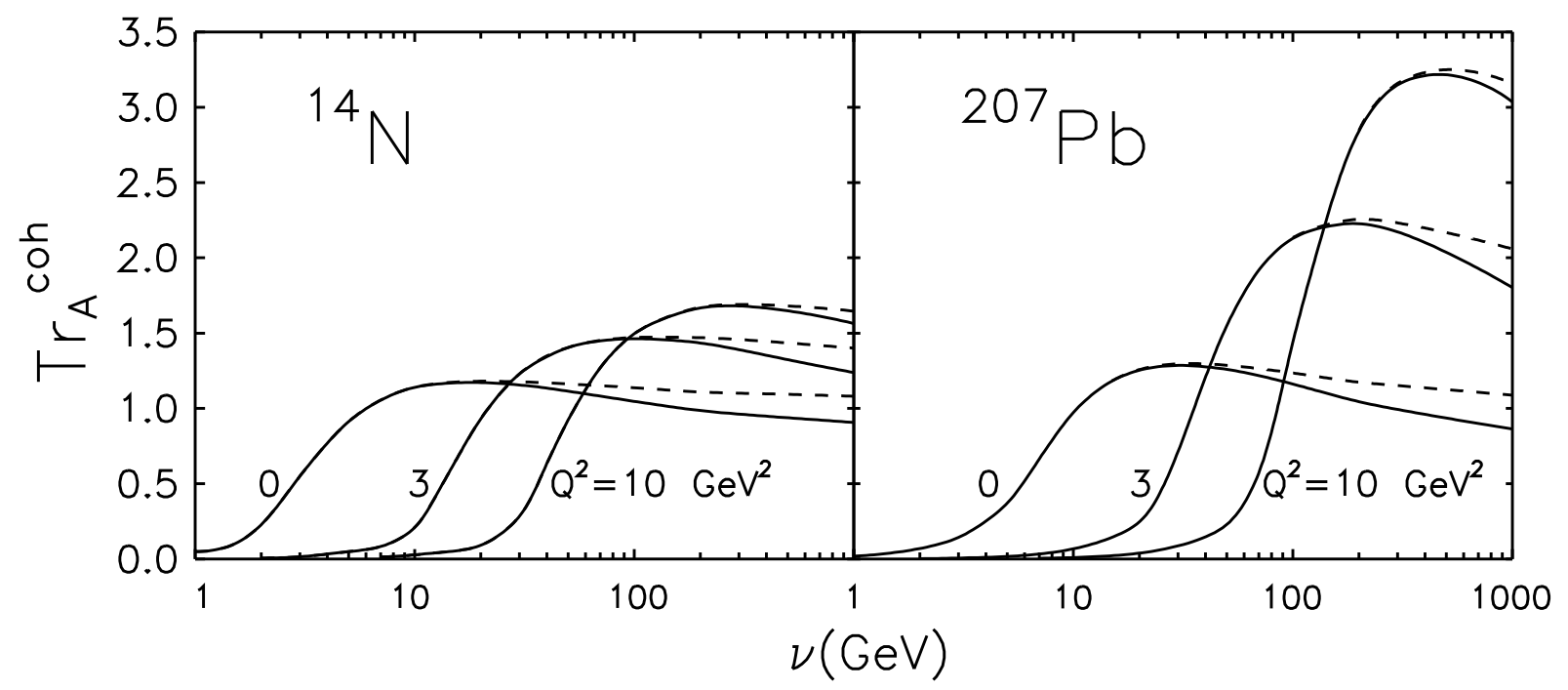

Figure 11: Nuclear transparency for incoherent electroproduction $\gamma^{*} A \rightarrow$ $\rho A$ as function of energy at $Q^{2}=0,3,10 \mathrm{GeV}^{2}$ for nitrogen and lead. The solid and dashed curves correspond to calculations with and without gluon shadowing, respectively.

nuclear matter is not transparent, but the nuclear coherent cross section is suppressed by the nuclear form factor. Indeed, the longitudinal momentum transfer which is equal to the inverse $\mathrm{CL}$, is large when the CL is short. However, at high energy $l_{c} \gg R_{A}$ and nuclear transparency nearly saturates (it decreases with $\nu$ only due to the rising dipole cross section). The saturation 
level is higher at larger $Q^{2}$ which is a manifestation of CT.

\subsection{Transverse momentum distribution}

Another manifestation of $\mathrm{CT}$ is a modification of the diffractive pattern in the momentum transfer dependence of the coherent cross section [55]. Indeed, the effect of CT on the nuclear transparency depends on impact parameter. Fourier transformation of such a modified amplitude will apparently result in a shifted positions of the diffractive minima. Indeed, calculations performed in the "frozen" approximation assuming sufficiently high energy $\left(l_{c} \gg R_{A}\right)$ lead to the $t$-dependence of nuclear transparency from Eq. (60) depicted in Fig. 12. We see

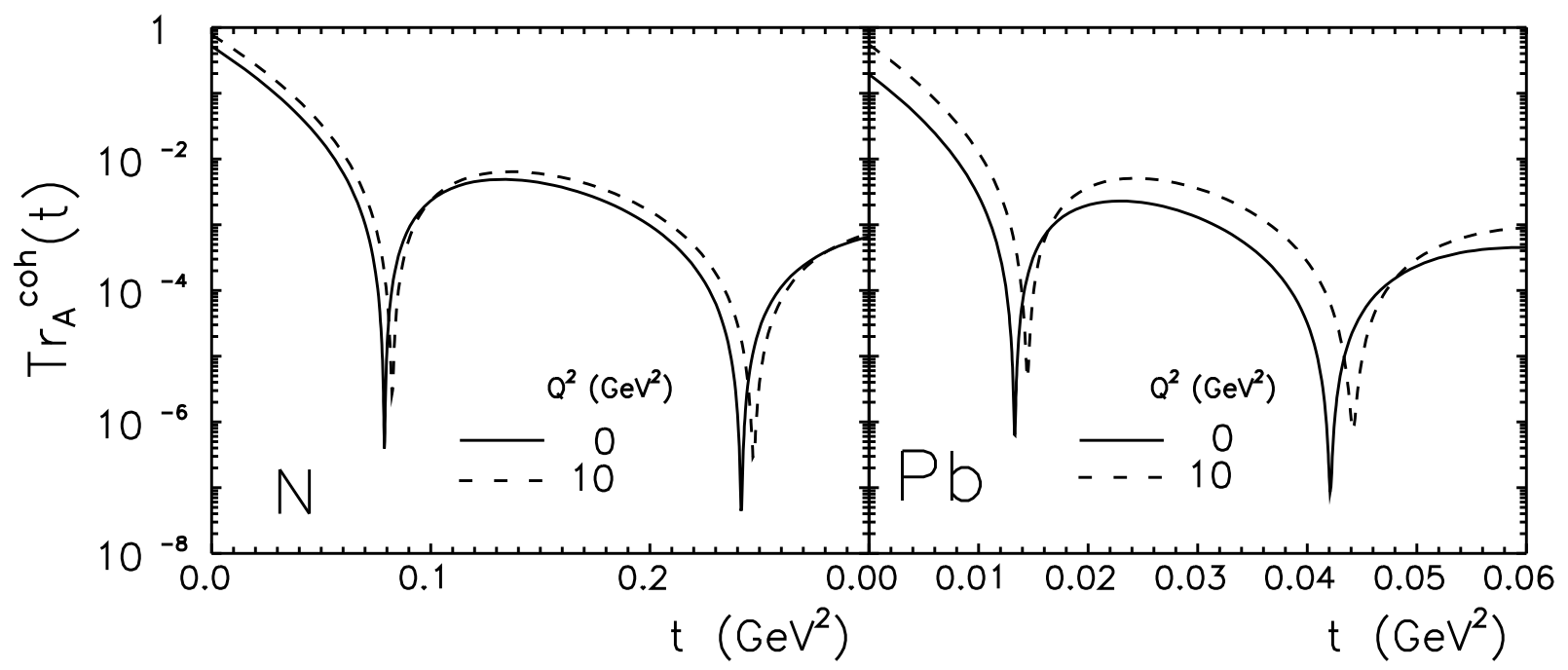

Figure 12: Nuclear transparency for coherent electroproduction $\gamma^{*} A \rightarrow$ $\rho^{0} A$ as function of momentum transfer squared calculated for nitrogen and lead in the limit of $l_{c} \gg R_{A}$. The solid and dashed curves correspond to $Q^{2}=0$ and $10 \mathrm{GeV}^{2}$ respectively.

that the CT effects shift the position of the diffractive minima to larger $t$. To understand the sign of the effect, we can use the approximate dipole cross section $\sigma_{\bar{q} q}(r)=C r^{2}$. Further, we can approximate the product of the photon and vector meson wave functions by a Gaussian $\propto \exp \left(-r^{2} /\left\langle r^{2}\right\rangle\right)$. The partial amplitude (amplitude for given impact parameter) of elastic 
production $\gamma^{*} \rightarrow \rho^{0}$ takes the form [2],

$$
\int d^{2} r \Psi_{V}^{*}(\vec{r}) \Psi_{\gamma^{*}}\left(\vec{r}, Q^{2}\right)\left\{1-\exp \left[-\frac{1}{2} C r^{2} T_{A}(b)\right]\right\}=\frac{C\left\langle r^{2}\right\rangle T_{A}(b)}{2+C\left\langle r^{2}\right\rangle T_{A}(b)} .
$$

The mean size $\left\langle r^{2}\right\rangle$ of the $\bar{q} q$ wave packet decreases with $Q^{2}$ suppressing the partial amplitude from Eq. (64). It follows from (64) the suppression is smaller on the periphery of the nucleus than in the center. This implies that the slope of the $t$-distribution $B_{\gamma^{*} A}=\left\langle b^{2}\right\rangle / 2$ should decrease with $Q^{2}$, i.e. the minima should move to larger values of $t$.

Although such a modification of the diffractive pattern should signal CT, the effect is very weak and its observation does not look feasible.

\section{Other pitfalls in the search for CT at low energies}

In order to avoid the effect of CL which leads for incoherent production to a nuclear transparency rising with $Q^{2}$ and mimics $\mathrm{CT}$ we suggested in Sect. 3.2 to study the $Q^{2}$ dependence in data samples which are preselected to have the same $l_{c}$. As soon as the CL effects are excluded, the Glauber model predicts no variation of the transparency with $Q^{2}$. There are, however, still other effects, not related to CT, which cause a growth of CT even if $l_{c}=$ const.

\subsection{Standard inelastic corrections}

The effect of CT can be treated in the hadronic representation as a multichannel problem [2, 66, 9]: the incident virtual photon produces diffractively on a bound nucleon either the ground state $V$, or any excitation. Only this stage of the process is $Q^{2}$ dependent. The produced states propagate further through the nucleus experiencing diagonal and off diagonal diffractive transitions. Eventually, the state $V$ is detected at macroscopic distances. These modifications of the Glauber single-channel approximation are at the heart of Gribov's inelastic shadowing.

The miracle of CT is the expectation that all those large amplitudes must cancel leaving only one amplitude, namely the direct production of the $V$. There is no hint from the hadronic representation that this should happen. We have no data for most of those amplitudes and no 
hope to measure them in future. Only the gauge invariance of QCD dictates this very nontrivial behavior which is not present in any of the old fashion models (colorless constituent quarks, etc.).

Nevertheless, we do have data for single diffraction which allow to calculate some of the lowest order inelastic corrections. Although these corrections are part of the whole CT phenomenon they are model independent (provided that those models are fitted to available data). In particular, the nuclear medium is known to be more transparent than expected using the Glauber model [2]. Indeed, if the produced $V$ state experiences inelastic diffraction inside the nucleus, it is gone from the detected channel according to the rules of the Glauber approximation. However, there is still a possibility to recover and come back to the $V$ channel in a subsequent collision, as is illustrated in Fig. 13. Apparently, this process increases the survival

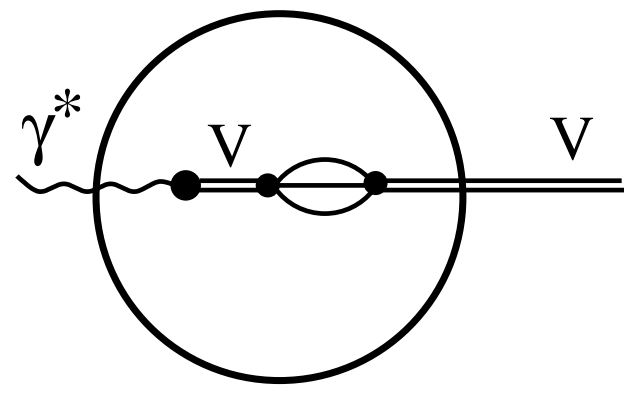

Figure 13: Intermediate diffractive excitation of a vector meson produced by the incident virtual photon and propagating through the nucleus.

probability for the $V$ state. There is clear experimental evidence that this takes place. The total cross sections of hadron- (neutrons, neutral kaons) nucleus interactions measured with high accuracy [67, 68] are smaller than the Glauber model predictions. This deviation increases with energy as it is controlled by the nuclear form factor which depends on the longitudinal momentum transfer $q_{L}$ in the diffraction dissociation.

Therefore, transparency of nuclear matter for hadrons increases with energy and this fact leads to a rising $Q^{2}$ dependence if data are selected according to the condition $l_{c}=$ const. 
Indeed, the energy rises according to the correlation $\nu=\left(Q^{2}+m_{V}^{2}\right) / 2 l_{c}$. Of course this effect is well known for the total cross sections since the pre-QCD era. It cannot be (and never was) interpreted as a manifestation of CT. Although these inelastic corrections are part of the CT phenomenon, one should admit that they exist independently of the answer to the question whether the CT is true or not.

Thus, one should be cautious in interpreting a rise of nuclear transparency as function of $Q^{2}$ at fixed $l_{c}$. The correction under discussion calculated in [69 for quasielastic high- $p_{T}$ electron scattering, $A\left(e, e^{\prime} p\right) X$ was found to be indistinguishable from the predicted CT effect up to rather high $Q^{2}$ of a few tens of $\mathrm{GeV}^{2}$.

The deviation of the transparency from the Glauber model prediction is calculated as 69],

$$
\begin{aligned}
\operatorname{Tr}_{A}^{i n c}\left(Q^{2}\right) & =\int d^{2} b \int_{-\infty}^{\infty} d z \rho_{A}(b, z) \exp \left[-\sigma_{i n}^{V N} \int_{z}^{\infty} d z^{\prime} \rho_{A}\left(b, z^{\prime}\right)\right] \\
& \times\left[1+\left.4 \pi \int d M^{2} \frac{d \sigma(V N \rightarrow X N)}{d M^{2} d t}\right|_{t=0} F_{A}\left(b, z, q_{L}\right)\right]^{2}
\end{aligned}
$$

Here $F_{A}\left(b, z, q_{L}\right)$ in (65) is the so called longitudinal form factor of the nucleus calculated at a given impact parameter $b$ and production coordinate $z$,

$$
F_{A}\left(b, z, q_{L}\right)=\int_{z}^{\infty} d z^{\prime} \rho_{A}\left(b, z^{\prime}\right) \cos \left(q_{L} z^{\prime}\right) .
$$

We use the same parameterization for the single diffraction cross section as in 67, 69] except for the normalization which is reduced by the factor $2 / 3$ as is suggested by the triple Regge phenomenology. Although it is a rather rough estimate, it is sufficient for our purpose, since the effect turns out to be very weak. The results of our calculations for the $Q^{2}$ dependence of the transparency are depicted by solid curves in Fig. 14 at fixed $l_{c}=1.35$ and $6.75 \mathrm{fm}$ (the bottom and upper curves respectively). One can see that although $\operatorname{Tr}_{A}^{\text {inc }}$ grows with $Q^{2}$, this effect when compared with Fig. 5 is too weak to be mixed up with the signal of CT. Indeed, the derivative $d \ln \left(\operatorname{Tr}_{A}^{i n c}\right) / d Q^{2}$ evaluated at $Q^{2}=1-2 \mathrm{GeV}^{2}$ equals 0.011 for nitrogen, 0.025 for krypton and 0.033 for lead. This is nearly an order of magnitude less than what was estimated in (56) as a signal for CT. 


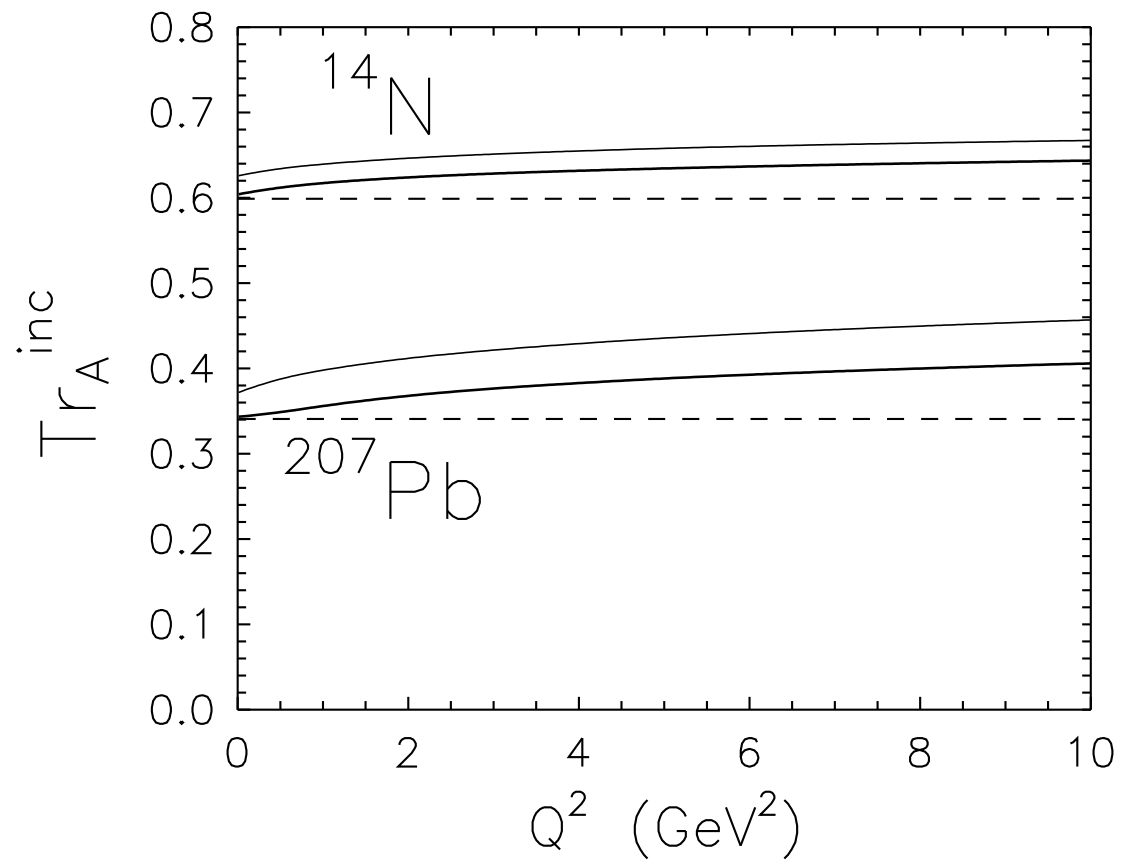

Figure 14: The same as in Fig. 5, but without any CT effects. Only the standard inelastic corrections which make the nuclear matter more transparent are included. The dashed curves correspond to the Glauber approximation. Each couple of solid curves correspond to $l_{c}=1.35 \mathrm{fm}$ (bottom curve) and $l_{c}=6.75 \mathrm{fm}$ (upper curve). The two sets of curves correspond to nitrogen (top) and lead (bottom)

\subsection{Finiteness of the $\rho$ meson lifetime}

Some of the effects have been calculated above at rather low energies when the lifetime of the $\rho$ is comparable with the nuclear size. For instance, at $\nu=2 \mathrm{GeV}$ the mean path length up to the decay is only $2.7 \mathrm{fm}$. Two pions have a smaller survival probability than the $\rho$ therefore the nuclear transparency should be smaller than is expected within the Glauber approximation disregarding decays. However, as function of energy the decay path length increases and eventually the nuclear transparency must reach the value corresponding to the Glauber model. Again, at $l_{c}=$ const the energy of $\rho$ rises with $Q^{2}$ and the nuclear transparency must grow with the decay length. This effect might cause a problem in identifying the signal of 
CT at low energy, and it should be considered with care. This correction is of less importance for $\phi$ production.

We corrected the Glauber formula for the finite decay length in the following way,

$$
\begin{aligned}
\operatorname{Tr}_{A}^{c o h} & =\gamma(\nu) \int d^{2} b \int_{-\infty}^{\infty} d z_{1} \rho_{A}(b, z) \int_{z_{1}}^{\infty} d z_{2} \\
& \times \exp \left[-\sigma_{i n}^{\rho N} \int_{z_{1}}^{z_{2}} d z^{\prime} \rho_{A}\left(b, z^{\prime}\right)-2 \sigma_{i n}^{\pi N} \int_{z_{2}}^{\infty} d z^{\prime} \rho_{A}\left(b, z^{\prime}\right)+\gamma(\nu)\left(z_{2}-z_{1}\right)\right]
\end{aligned}
$$

where

$$
\gamma(\nu)=\frac{\Gamma_{\rho} m_{\rho}}{\sqrt{\nu^{2}-m_{\rho}^{2}}},
$$

is the Lorentz enhanced decay length of the $\rho$ meson, $\Gamma_{\rho}=0.15 \mathrm{GeV}$ is the total decay width of the $\rho$. Like previously, $\nu$ correlates with $Q^{2}$ via Eq. (54).

The results of our calculations are shown in Fig. 15 as function of $Q^{2}$ at different fixed $l_{c}$. The effect turns out to be very weak compared to the expected effect of CT demonstrated in Fig. 5. For a CL of $l_{c}=1.35 \mathrm{fm}(6.75 \mathrm{fm})$ the results are shown by the bottom (upper) solid curves for both nuclei. The derivative $d \ln \left(\operatorname{Tr}_{A}^{i n c}\right) / d Q^{2}$ evaluated at $Q^{2}=1-2 \mathrm{GeV}^{2}$ equals $0.014(0.003)$ and $0.03(0.008)$ for nitrogen and lead respectively. We conclude that the effect of the finite $\rho$ decay length cannot be mixed up with a CT signal.

It is disputable whether the two pions emerging from a $\rho$ decay immediately starts to attenuate with twice the pion absorption cross section. One might think about two pions which strongly overlap at the production point, then their cross section should be reduced due to color screening. This is a general problem of how one should decide whether decay has already happened or not. It is easier to understand for the example of photon radiation by an electron. One can treat the photon as being originally a part of the static Coulomb field of the electron which is then shaken off by the interaction with a target. Only when the photon and electron become incoherent they start acting as independent partons. It takes, however, a time span dictated by the uncertainty principle to discriminate between a coherent system, electron and its field, and an incoherent pair of an electron plus a photon. This time is called radiation or coherence time. In analogy, one can say that the $\rho$ meson has already decayed when the 


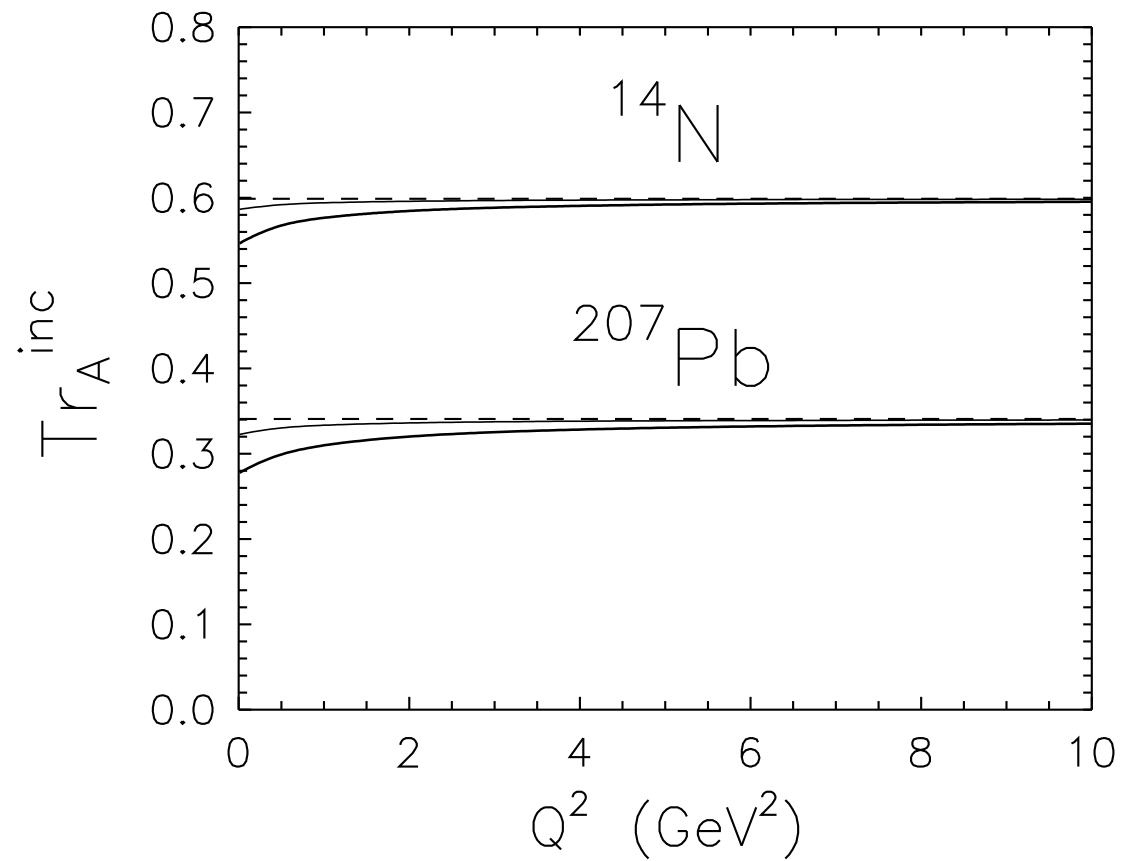

Figure 15: The same as in Figs. 囵 and 14, but with no effects of either $C T$ or any inelastic correction included. Only the finite $\rho$ meson decay length which leads to an enhanced absorption is taken into account. For each nucleus the dashed curve corresponds to the Glauber approximation $\left(\Gamma_{\rho} \rightarrow 0\right)$, the bottom and upper solid curves correspond to $l_{c}=1.35$ and $6.75 \mathrm{fm}$ respectively.

two pions become incoherent. In this case they interact independently and Eq. (67) is valid. However, while the pions are still coherent, one should treat them as intrinsic components of the $\rho$ meson.

\section{Gluon shadowing}

At very small $x_{B j}$ the density of gluons should eventually deviate from the predicted by the DGLAP evolution (as we mentioned, whether an indication of this saturation effect was already seen at HERA is controversial). In the infinite momentum frame this phenomenon looks like 
gluon-gluon fusion. It corresponds to a nonlinear term in the evolution equation [70]. This effect should lead to a suppression of small- $x_{B j}$ gluons in a nucleon.

One may expect a precocious onset of the saturation effects for heavy nuclei. In the infinite momentum frame of the nucleus the gluon clouds of nucleons which have the same impact parameter overlap at small $x_{B j}$ in longitudinal direction. This allows gluons originated from different nucleons to fuse leading to a gluon density which is not proportional to the density of nucleons any more. This is gluon shadowing.

Such a parton model interpretation is not Lorentz invariant, the same phenomenon looks quite different in the rest frame of the nucleus. It corresponds to the process of gluon radiation and shadowing corrections related to multiple interactions of the radiated gluons in the nuclear medium [19]. This is a coherence phenomenon known as the Landau-Pomeranchuk effect, namely the suppression of bremsstrahlung by interference of radiation from different scattering centers. It demands a sufficiently long coherence time of radiation, a condition equivalent to demanding a small Bjorken $x_{B j}$ in the parton model.

Although the two interpretations look so different, one can get a hint that they are the same phenomenon relating them to the Reggeon graphs which are Lorentz invariant. The doublescattering correction to the cross section of gluon radiation depicted in Fig. 16a corresponds to the absorptive part of elastic $p A$ amplitude shown in Fig. 16b. Since the initial and final nucleons are colorless, each pair of exchanged gluons attached to the same nucleon is in a colorless state, i.e. represents the Pomeron (in the Born approximation). Thus, the Feynman graph in Fig. 16b is a part of the triple-Pomeron diagram shown in Fig. 16c. It can be interpreted as fusion of two Pomerons originated from different nucleons, 2IP $\rightarrow \mathbb{P}$. This observation bridges the two interpretations of gluon shadowing.

Note that in the hadronic representation such a suppression of parton density corresponds to Gribov's inelastic shadowing [1] which is related to the single diffraction cross section. In particular, gluon shadowing corresponds to the triple-Pomeron term in the diffractive dissociation cross section which enters the calculations of inelastic corrections (Fig. 16. $)$.

There are still very few numerical evaluations of gluon shadowing in the literature, all done 


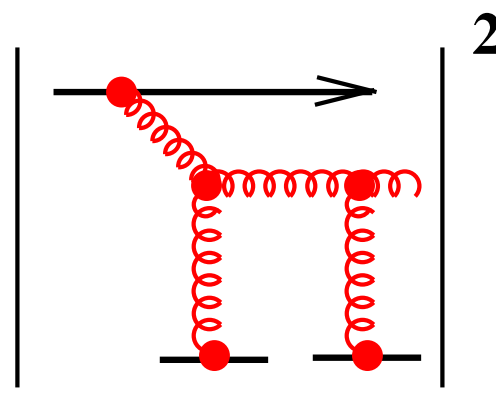

a

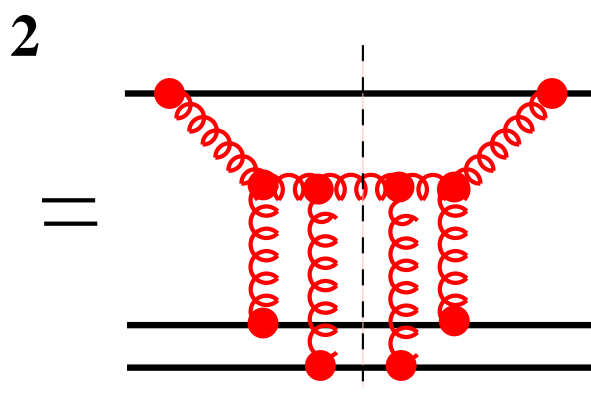

b

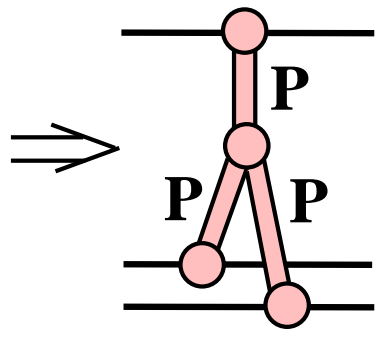

c

Figure 16: The double scattering correction to gluon radiation in the rest frame of the target nucleus (a). The absorptive part of the corresponding elastic $p A$ amplitude (b). The triple-Pomeron graph representing fusion $2 \mathbb{P} \rightarrow \mathbb{P}(\mathbf{c})$.

in the rest frame of the nucleus using the idea from [19]. It turns out that even at low $Q^{2}$ in the nonperturbative domain where one should expect the strongest shadowing effects they are rather weak. Indeed, data for diffractive excitation of the incident hadrons to the states of large mass, the so called triple-Pomeron region, show that the cross section is amazingly small, an order of magnitude smaller than one could expect by comparing with the cross section of small mass excitation [18]. To explain such a smallness one has to assume a rather small radius of propagation of the LC gluons, $r_{0} \approx 0.3 \mathrm{fm}[23$, 71]. It is clear that such a small quark-gluon fluctuation also leads to a rather weak gluon shadowing.

To incorporate the smallness of the size of quark-gluon fluctuations into the LC dipole approach a nonperturbative LC potential describing the quark-gluon interaction was introduced into the Schrödinger equation for the LC Green function describing the propagation of a quarkgluon system. The strength of the potential was fixed by data on high mass $\left(M_{X}^{2}\right)$ diffraction $p p \rightarrow p X$ [23]. This approach allows to extend the methods of pQCD to the region of small $Q^{2}$. Since a new semihard scale $1 / r_{0} \sim 0.65 \mathrm{GeV}$ is introduced one should not expect a substantial variation of gluon shadowing at $Q^{2} \lesssim 4 / r_{0}^{2}$. Indeed, calculations performed in [23] for $Q^{2}=0$ and $4 \mathrm{GeV}^{2}$ using different techniques led to about the same gluon shadowing. At higher $Q^{2}$ shadowing slowly (logarithmically) decreases in accordance with expectations based on the 
evolution equation [72].

We repeated the calculations [23] of the ratio of the gluon densities in nuclei and nucleon,

$$
R_{G}\left(x_{B j}, Q^{2}\right)=\frac{G_{A}\left(x_{B j}, Q^{2}\right)}{A G_{N}\left(x_{B j}, Q^{2}\right)} \approx 1-\frac{\Delta \sigma(\bar{q} q G)}{\sigma_{t o t}^{\gamma^{*} A}},
$$

where $\Delta \sigma(\bar{q} q G)$ is the inelastic correction to the total cross section $\sigma_{\text {tot }}^{\gamma^{*} A}$ related to the creation of a $\bar{q} q G$ intermediate state,

$$
\begin{gathered}
\Delta \sigma(\bar{q} q G)=\operatorname{Re} \int_{-\infty}^{\infty} d z_{2} \int_{-\infty}^{z_{2}} d z_{1} \rho_{A}\left(b, z_{1}\right) \rho_{A}\left(b, z_{2}\right) \int d^{2} x_{2} d^{2} y_{2} d^{2} x_{1} d^{2} y_{1} \int d \alpha_{q} \frac{d \alpha_{G}}{\alpha_{G}} \\
\times \quad F_{\gamma^{*} \rightarrow \bar{q} q G}^{\dagger}\left(\vec{x}_{2}, \vec{y}_{2}, \alpha_{q}, \alpha_{G}\right) G_{\bar{q} q G}\left(\vec{x}_{2}, \vec{y}_{2}, z_{2} ; \vec{x}_{1}, \vec{y}_{1}, z_{1}\right) F_{\gamma^{*} \rightarrow \bar{q} q G}\left(\vec{x}_{1}, \vec{y}_{1}, \alpha_{q}, \alpha_{G}\right) .
\end{gathered}
$$

Here $\vec{x}$ and $\vec{y}$ are the transverse distances from the gluon to the quark and antiquark, respectively. $\alpha_{q}$ is the fraction of the LC momentum of the $\bar{q} q$ carried by the quark, and $\alpha_{G}$ is the fraction of the photon momentum carried by the gluon. $F_{\gamma^{*} \rightarrow \bar{q} q G}$ is the amplitude of diffractive $\bar{q} q G$ production in a $\gamma^{*} N$ interaction [23],

$$
\begin{aligned}
F_{\gamma^{*} \rightarrow \bar{q} q G}\left(\vec{x}, \vec{y}, \alpha_{q}, \alpha_{G}\right) & =\frac{9}{8} \Psi_{\bar{q} q}\left(\alpha_{q}, \vec{x}-\vec{y}\right)\left[\Psi_{q G}\left(\frac{\alpha_{G}}{\alpha_{q}}, \vec{x}\right)-\Psi_{\bar{q} G}\left(\frac{\alpha_{G}}{1-\alpha_{q}}, \vec{y}\right)\right] \\
& \times\left[\sigma_{\bar{q} q}(x)+\sigma_{\bar{q} q}(y)-\sigma_{\bar{q} q}(\vec{x}-\vec{y})\right]
\end{aligned}
$$

where $\Psi_{\bar{q} q}$ and $\Psi_{\bar{q} G}$ are the LC distribution functions of the $\bar{q} q$ fluctuations of a photon and $q G$ fluctuations of a quark, respectively.

$G_{\bar{q} q G}\left(\vec{x}_{2}, \vec{y}_{2}, z_{2} ; \vec{x}_{1}, \vec{y}_{1}, z_{1}\right)$ is the LC Green function which describes propagation of the $\bar{q} q G$ system from the initial state with longitudinal and transverse coordinates $z_{1}$ and $\vec{x}_{1}, \vec{y}_{1}$, respectively, to the final coordinates $\left(z_{2}, \vec{x}_{2}, \vec{y}_{2}\right)$. For the calculation of gluon shadowing one should suppress the intrinsic $\bar{q} q$ separation, i.e. to assume $\vec{x}=\vec{y}$. In this case the Green function essentially simplifies and describes propagation of a gluon-gluon dipole through a medium.

An important finding of Ref. [23] is the presence of a strong nonperturbative interaction which squeezes the gluon-gluon wave packet and substantially diminishes gluon shadowing. The smallness of the gluon-gluon transverse separation is not a model assumption, but is dictated by data for hadronic diffraction to large masses (triple-Pomeron regime) which is controlled by diffractive gluon radiation. 
Further calculational details can be found in [23]. Here we calculate $R_{G}$ [Eq. (69)] for different nuclear thicknesses $T_{A}(b)$. Since we use an approximation of constant nuclear density (see Appendix A),$T_{A}(b)=\rho_{0} L$, where $L=2 \sqrt{R_{A}^{2}-b^{2}}$, the ratio $R_{G}\left(x_{B j}, Q^{2}\right)$ is also implicitly a function of $L$. An example for the calculated $L$-dependence of $R_{G}\left(x_{B j}, Q^{2}\right)$ at $Q^{2}=4 \mathrm{GeV}^{2}$ is depicted in Fig. 17 for different values of $x_{B j}$. As one should expect, the longer $L$, the stronger

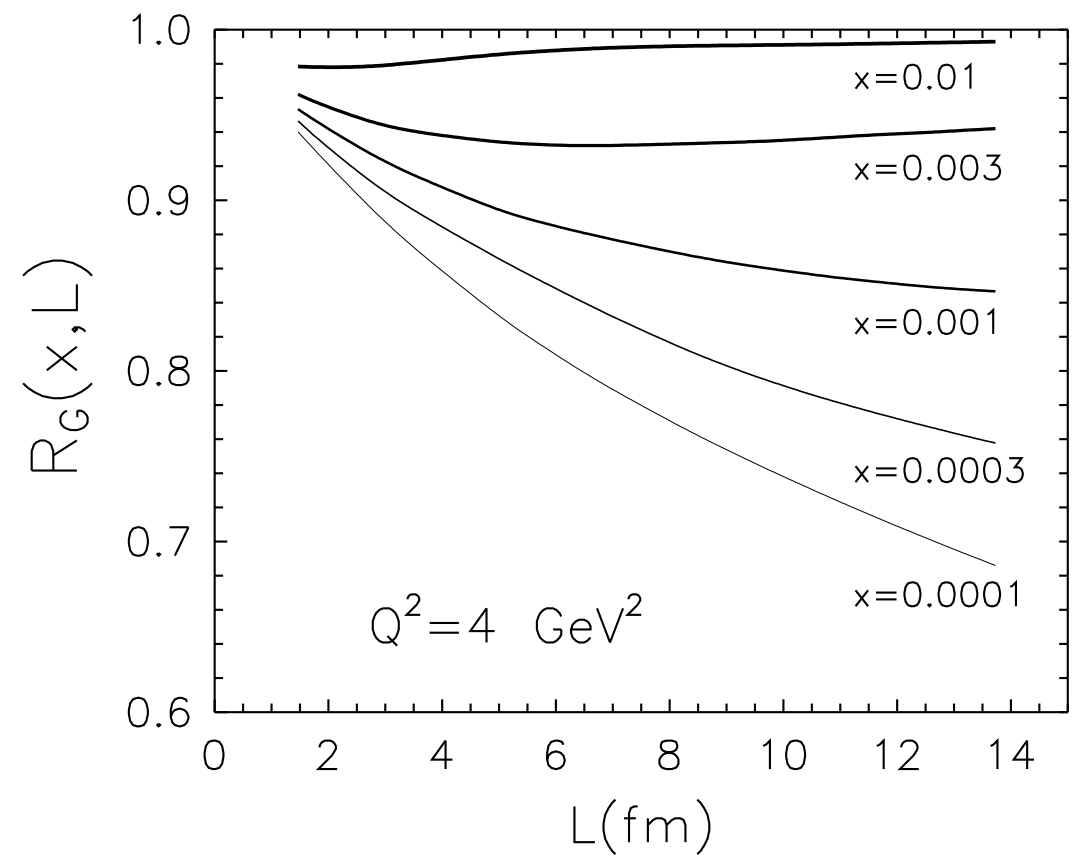

Figure 17: The ratio of nucleus to nucleon gluon densities as function of the thickness of the nucleus, $L=T(b) / \rho_{0}$, at $Q^{2}=4$ and different fixed values of $x_{B j}$.

is gluon shadowing at small $x_{B j}$.

We calculated the gluon shadowing only for the lowest Fock component containing just one LC gluon. In terms of the parton model it reproduces the effects of fusion of many gluons to one gluon (in terms of Regge approach it corresponds to $n \mathbb{P} \rightarrow \mathbb{P}$ vertex). Inclusion of higher multigluon Fock components is still a challenge. However, their effect can be essentially taken into account by eikonalization of the calculated $R_{G}\left(x_{B j}, Q^{2}\right)$, as argued in [73]. In other words, the dipole cross section which is proportional to the gluon density at small separations, should 
be renormalized everywhere,

$$
\sigma_{\bar{q} q} \Rightarrow R_{G} \sigma_{\bar{q} q} .
$$

Such a procedure makes the nuclear medium more transparent. This could be expected since Gribov's inelastic shadowing is known to suppress the total hadron-nucleus cross sections, i.e. to make nuclei more transparent [2, 69].

It is interesting, that the cross section of incoherent electroproduction of vector mesons is rather insensitive to gluon shadowing. Indeed, although the renormalization Eq. (72) suppresses the pre-exponential factor $\sigma_{\bar{q} q}(r, s)$ on the r.h.s. of Eq. (51), it simultaneously increases the exponential. These two effects essentially cancel. Indeed, our predictions for the effect of gluon shadowing for incoherent production $\gamma^{*} A \rightarrow \rho^{0} X$ depicted in Fig. 8 demonstrate a rather small difference between the curves with (solid) and without (dashed) gluon shadowing.

A few observations are in order. First, the onset of gluon shadowing happens at rather high energy $\nu>100 \mathrm{GeV}$. This corresponds to the claim made in 23] that the onset of gluon shadowing requires smaller $x_{B j}$ than the onset of quark shadowing. This is because the fluctuations containing gluons are in general heavier than the $\bar{q} q$ and have a shorter CL.

Then, one can see that a stronger effect of gluon shadowing is expected for nitrogen than for lead. Although it contradicts simple intuition, it is easy interpreted. The renormalization of the dipole cross section Eq. (72) may either suppress or enlarge the incoherent cross section in Eq. (51) depending on the value of the nuclear thickness function $T(b)$. Namely, it should lead to a suppression for small $T(b)$, but to an enhancement for large $T(b)$. Indeed we observe this trend in Fig. 8. Some enhancement (antishadowing) can be seen for lead at $Q^{2}=0$. The results presented for nitrogen show that the effect is maximal at intermediate values of $Q^{2}$ while it is smaller at $Q^{2}=0$ and $10 \mathrm{GeV}$. This is a result of the same interplay between the pre-exponential factor and the exponential in Eq. (51).

The implication of gluon shadowing for the case of coherent production $\gamma^{*} A \rightarrow V A$ is clearer. It is easy to understand that it always diminishes the coherent cross section. In terms of VDM the photoproduction cross section is related to the total $V A$ cross section which is 
always reduced by inelastic corrections. One can also see from Eq. (62) that the suppression of the dipole cross section by the renormalization [Eq. (72)] can result only in a reduction of the cross section. Thus, we expect much stronger effects of gluon shadowing for coherent than for incoherent production. The results of calculations based on the exact expression Eq. (61) confirm this conjecture. The predicted suppression of the coherent cross section is stronger for heavy nuclei and low $Q^{2}$.

\section{Summary and conclusions}

Electroproduction of vector mesons off nuclei is subject to an interplay between coherence (shadowing) and formation (color transparency) effects. Conventionally, one can associate those effects with the initial and final state interaction respectively. We developed a rigorous quantum-mechanical approach based on the light-cone QCD Green function formalism which naturally incorporates these interference effects. Our main results and observations are the following.

- The suggested approach allows to find for the first time a comprehensive model for the long standing problem of exclusive electroproduction of vector mesons off nuclei. The main result of the paper, Eq. (52), interpolates between the previously known low and high energy limits for incoherent production. Eq. (58) does the same for coherent production.

- The onset of coherence effects (shadowing) can mimic the expected signal of CT in incoherent electroproduction of vector mesons at medium and low energies. In order to single out the formation effect data must be binned in $l_{c}$ and $Q^{2}$. Observation of a rising nuclear transparency as function of $Q^{2}$ for fixed $l_{c}$ would signal color transparency.

- Due to quark-hadron duality the Green function formalism under consideration is equivalent to a solution of the full multi-channel problem in the hadronic representation. We found a much steeper $Q^{2}$ dependence of nuclear transparency (Fig. 5), i.e. a stronger signal of CT, than was predicted in [9] within the two-channel approximation. Moreover, 
the slope of the $Q^{2}$ dependence is even higher at lower energies. This should allow to detect a signal of CT in experiments with the HERMES spectrometer and especially at JLab.

- The successful experimental confirmation [64] of the predicted coherence length effects [16] seems now to be a accidental consequence of the specific correlation between $Q^{2}$ and $l_{c}$ in the HERMES data. The present parameter-free calculations well describe the observed variation of nuclear transparency with $l_{c}$ (Fig. 7) as a result of a complicated interplay between the effects of CT and coherence length.

- There are other effects which may cause a rise of nuclear transparency with $Q^{2}$ at $l_{c}=$ const thus mimicking a signal of CT. These are the lowest order inelastic corrections which are rather precisely fixed by available data for diffraction, and the finite lifetime of vector mesons. Both effects lead to the more transparent nuclear medium at higher energies, i.e. at larger $Q^{2}$ due to the correlation in Eq. (54) between $\nu$ and $Q^{2}$. We found, however, both effects to be too weak (Figs. 14 and 15) to be relevant.

- The effects of CT in coherent production of vector mesons are found to be less pronounced. Although transparency decreases with $Q^{2}$ and does not mimic CT in this case, the CL effects significantly modify the $Q^{2}$ dependence and may completely eliminate any signal of CT at medium energies. Besides, the cross section of coherent production is very small at low energies (Fig. 11).

- The effects of CT modify the impact-parameter dependence of the amplitude of coherent production by diminishing the mean square of the impact parameter in the interaction amplitude. Therefore the positions of the diffractive minima in the differential cross section are expected to shift to larger values of $|t|$ (Fig. 12). However the effects seems to be too small to be reliably observed.

- Although it has been known how to calculate nuclear transparency in the high energy limit $l_{c} \gg R_{A}$ [8, 57], the effect of gluon shadowing was missed. We calculated nuclear 
suppression of gluons at small $x_{B j}$ within the same LC approach treating it as shadowing corrections for the higher Fock states containing gluons. The nonperturbative interaction of the LC gluons significantly reduces the predicted magnitude of gluon shadowing (Fig. 17). Although the amplitude of meson production off a bound nucleon is suppressed due to a reduced amount of gluons in the nucleus, the same effect makes the nuclear medium more transparent and enhances the meson survival probability. For incoherent $\rho$ production these two effects nearly compensate each other for heavy nuclei (Fig. 8). The cross section for coherent production is less for more transparent nuclei, therefore the effect of gluon shadowing is more pronounced (Fig. 11). These corrections are not important at HERMES or JLab energies, but are significant at the higher energies of eRHIC and for coherent Coulomb production in heavy ion collisions at RHIC.

Concluding, the predicted large effects of $\mathrm{CT}$ in incoherent electroproduction of vector mesons off nuclei open new possibilities for the search for CT with medium energy electrons.

Acknowledgment: We are grateful to Alexander Borissov and Kawtar Hafidi for inspiring

questions. J.N. is thankful to B. Povh for hospitality at Max-Planck-Institut für Kernphysik where this paper was finished. This work has been partially supported by a grant from the Gesellschaft für Schwerionenforschung Darmstadt (GSI), grant No. GSI-OR-SCH, and by the European Network: Hadronic Physics with Electromagnetic Probes, Contract No. FMRXCT96-0008. The work of J.N. has been supported in part by the Slovak Funding Agency, Grant No. 2/1169 and Grant No. 6114.

\section{Appendix A Approximation for the dipole cross section}

To keep the calculations simple we are forced to use the approximate dipole cross section Eq. (46) which allows to obtain the Green function in an analytical form as is described in Sect. 3.1. We fix the factor $C(s)$ by demanding that calculations employing the approximation Eq. (46) reproduce correctly the results based on the realistic cross section in the limit $l_{c} \gg R_{A}$ 
when the Green function takes the simple form (47). Thus, for incoherent production of vector mesons the factor $C(s)$ is fixed by the relation,

$$
\begin{aligned}
& \frac{\int d^{2} b T_{A}(b)\left|\int d^{2} r r^{2} \exp \left[-\frac{1}{2} C_{T, L}(s) r^{2} T_{A}(b)\right] \int d \alpha \Psi_{V}^{*}(\vec{r}, \alpha) \Psi_{\gamma^{*}}^{T, L}(\vec{r}, \alpha)\right|^{2}}{\left|\int d^{2} r r^{2} \int d \alpha \Psi_{V}^{*}(\vec{r}, \alpha) \Psi_{\gamma^{*}}^{T, L}(\vec{r}, \alpha)\right|^{2}} \\
= & \frac{\int d^{2} b T_{A}(b)\left|\int d^{2} r \sigma_{\bar{q} q}(r, s) \exp \left[-\frac{1}{2} \sigma_{\bar{q} q}(r, s) T_{A}(b)\right] \int d \alpha \Psi_{V}^{*}(\vec{r}, \alpha) \Psi_{\gamma^{*}}^{T, L}(\vec{r}, \alpha)\right|^{2}}{\left|\int d^{2} r \sigma_{\bar{q} q}(r, s) \int d \alpha \Psi_{V}^{*}(\vec{r}, \alpha) \Psi_{\gamma^{*}}^{T, L}(\vec{r}, \alpha)\right|^{2}}
\end{aligned}
$$

Correspondingly, for coherent production the factor $C(s)$ is fixed by the relation,

$$
\begin{aligned}
& \frac{\int d^{2} b\left|\int d^{2} r \int d \alpha \Psi_{V}^{*}(\vec{r}, \alpha) \Psi_{\gamma^{*}}^{T, L}(\vec{r}, \alpha)\left\{1-\exp \left[-\frac{1}{2} C_{T, L}(s) r^{2} T_{A}(b)\right]\right\}\right|^{2}}{\left|\int d^{2} r \int d \alpha \Psi_{V}^{*}(\vec{r}, \alpha) C_{T, L}(s) r^{2} \Psi_{\gamma^{*}}^{T, L}(\vec{r}, \alpha)\right|^{2}} \\
= & \frac{\int d^{2} b\left|\int d^{2} r \int d \alpha \Psi_{V}^{*}(\vec{r}, \alpha) \Psi_{\gamma^{*}}^{T, L}(\vec{r}, \alpha)\left\{1-\exp \left[-\frac{1}{2} \sigma_{\bar{q} q}(r, s) T_{A}(b)\right]\right\}\right|^{2}}{\left|\int d^{2} r \int d \alpha \Psi_{V}^{*}(\vec{r}, \alpha) \sigma_{\bar{q} q}(r, s) \Psi_{\gamma^{*}}^{T, L}(\vec{r}, \alpha)\right|^{2}},
\end{aligned}
$$

To take advantage of the analytical form of the Green function which is known only for the LC potential Eq. (45) with a constant nuclear density, we use the approximation $\rho_{A}(b, z)=$ $\rho_{0} \Theta\left(R_{A}^{2}-b^{2}-z^{2}\right)$. Therefore we have to use this form for Eqs. (A.1) and (A.2) as well. The value of the mean nuclear density $\rho_{0}$ has been determined using the relation,

$$
\int d^{2} b\left[1-\exp \left(-\sigma_{0} \rho_{0} \sqrt{R_{A}^{2}-b^{2}}\right)\right]=\int d^{2} b\left[1-\exp \left(-\frac{\sigma_{0}}{2} T(b)\right)\right],
$$

where the nuclear thickness function $T_{A}(b)$ is calculated with the realistic Wood-Saxon form of the nuclear density. The value of $\rho_{0}$ turns out to be practically independent of the cross section $\sigma_{0}$ in the range from 1 to $50 \mathrm{mb}$.

\section{References}

[1] V.N. Gribov, Sov. Phys. JETP 29, 483 (1969) [Zh. Eksp. Teor. Fiz. 56, 892 (1969)].

[2] A.B. Zamolodchikov, B.Z. Kopeliovich and L.I. Lapidus, Pis'ma Zh. Eksp. Teor. Fiz. 33, 612 (1981); Sov. Phys. JETP Lett. 33, 595 (1981). 
[3] G. Bertsch, S.J. Brodsky, A.S. Goldhaber and J.F. Gunion, Phys. Rev. Lett. 47, 297 (1981).

[4] P. Jain, B. Pire and J.P. Ralston, Phys. Rept. 271, 67 (1996).

[5] J.F. Gunion and D.E. Soper, Phys. Rev. D15, 2617 (1977).

[6] J. Hüfner and B. Povh, Phys. Rev. D46, 990 (1992).

[7] B. Povh, Hadron Interactions - Hadron Sizes, hep-ph/9806379

[8] B.Z. Kopeliovich and B.G. Zakharov, Phys. Rev. D44, 3466 (1991).

[9] J. Hüfner and B.Z. Kopeliovich, Phys. Lett. B403, 128 (1997).

[10] T.H. Bauer et al., Rev. Mod. Phys. 50, 261 (1978).

[11] The NMC Collaboration, M. Arneodo et al., Nucl. Phys. 441, 12 (1995); Nucl. Phys. 481, 3 (1996).

[12] The E665 Collaboration, M.R. Adams et al., Z. Phys. C67, 403 (1995).

[13] B.Z. Kopeliovich, Phys. Lett. B227, 461 (1989).

[14] The E772 Collaboration, D.M. Alde et al., Phys. Rev. Lett. 64, 2470 (1990).

[15] The E866 Collaboration, M. Vasiliev et al., Phys. Rev. Lett. 83, 2304 (1999).

[16] J. Hüfner, B.Z. Kopeliovich and J. Nemchik, Phys. Lett. B383, 362 (1996).

[17] V. Karmanov and L.A. Kondratyuk, Sov. Phys. JETP Lett. 18, 266 (1973).

[18] Yu.M. Kazarinov, B.Z. Kopeliovich, L.I. Lapidus and I.K. Potashnikova, JETP 70, 1152 (1976).

[19] A.H. Mueller, Nucl. Phys. B335, 115 (1990).

[20] B.Z. Kopeliovich, J. Raufeisen and A.V. Tarasov, Phys. Rev. C62, 035204 (2000). 
[21] E. Gotsman, E. Levin and U. Maor, Phys. Lett. 425, 369 (1998)

[22] K. Golec-Biernat and M. Wüsthoff, Phys. Rev. D59, 014017 (1999); Phys. Rev. D60, $114023(1999)$.

[23] B.Z. Kopeliovich, A. Schäfer and A.V. Tarasov, Phys. Rev. D62, 054022 (2000).

[24] A. Donnachie and P.V. Landshoff, Phys. Lett. B478, 146 (2000).

[25] Review of Particle Physics, Particle Data Group, Eur. Phys. J. C15, 1 (2000).

[26] V.N. Gribov, Sov. J. Nucl. Phys. 9, 369 (1969) [Yad. Fiz. 9, 640 (1969)].

[27] J.B. Bronzan, G.L. Kane and U.P. Sukhatme, Phys.Lett. B49 (1974) 272.

[28] J.B. Kogut and D.E. Soper, Phys. Rev. D 1, 2901 (1970).

[29] J.M. Bjorken, J.B. Kogut and D.E. Soper, Phys. Rev. D 3, 1382 (1971).

[30] N.N. Nikolaev and B.G. Zakharov, Z. Phys. C49, 607 (1991).

[31] R.P. Feynman and A.R. Gibbs, Quantum Mechanics and Path Integrals, McGraw-Hill Book Company, NY 1965.

[32] H1 Collaboration, S. Aid et al., Z. Phys. C69, 27 (1995).

[33] ZEUS Collaboration, M. Derrick et al., Phys. Lett. B293, 465 (1992).

[34] M.V. Terent'ev, Sov. J. Nucl. Phys. 24, 106 (1976).

[35] I. Halperin and A. Zhitnitsky, Phys. Rev. D56, 184 (1997).

[36] J. Nemchik, N.N. Nikolaev, E. Predazzi and B.G. Zakharov, Z. Phys. C75, 71 (1997).

[37] NMC Collaboration, M. Arneodo et al., Nucl. Phys. B429, 503 (1994).

[38] H1 Collaboration, C. Adloff et al., Eur. Phys. J. C13, 371 (2000). 
[39] ZEUS Collaboration, J. Breitweg et al., Eur. Phys. J. C6, 603 (1999).

[40] H1 Collaboration, C. Adloff et al., Z. Phys. C75, 607 (1997);

H1 Collaboration, C. Adloff et al., Phys. Lett B483, 360 (2000);

ZEUS Collaboration, M. Derrick et al., Phys. Lett. B380, 220 (1996);

ZEUS Collaboration, J. Breitweg et al., "Exclusive Electroproduction of $\Phi$ Mesons at HERA", presented at the XXIX International Conference on HEP, Vancouver, 1998, paper793.

[41] J. Nemchik, N.N. Nikolaev, E. Predazzi, B.G. Zakharov and V.R. Zoller, J. Exp. Theor. Phys. 86, 1054 (1998).

[42] CHIO Collaboration, W.D. Shambroom et al., Phys. Rev. D26, 1 (1982);

E665 Collaboration, M.R. Adams et al., Z. Phys. C74, 237 (1997).

[43] H1 Collaboration, S. Aid et al., Nucl. Phys. B463, 3 (1996).

[44] H1 Collaboration, S. Aid et al., Nucl. Phys. B468, 3 (1996).

[45] ZEUS Collaboration, M. Derrick et al., Z. Phys. C69, 39 (1995).

[46] ZEUS Collaboration, J. Breitweg et al., Z. Phys. C73, 253 (1997);

ZEUS Collaboration, J. Breitweg et al., Eur. Phys. J. C2, 247 (1998).

[47] ZEUS Collaboration, M. Derrick et al., Phys. Lett. B356, 601 (1995).

[48] J. Busenitz et al.,Phys. Rev. D40, 1 (1989), and references therein;

R. Erbe et al., Phys. Rev. 175, 1669 (1968);

J. Ballam et al., Phys. Rev. D7, 3150 (1973);

H.J. Bersh et al., Nucl. Phys. B70, 257 (1974);

H.J. Behrend et al., Phys. Lett. B56, 408 (1975);

D.P. Barber et al., ibid. B79, 150 (1978); 
D. Aston et al., Nucl. Phys. B172, 1 (1980);

M. Atkinson et al., Z. Phys. C27, 233 (1985).

[49] ZEUS Collaboration, M. Derrick et al., Phys. Lett. B377, 259 (1996).

[50] OMEGA Collaboration, D. Aston et al., Nucl. Phys. B209, 56 (1982).

[51] J. Park et al., Nucl. Phys. B36, 404 (1972);

R.M. Egloff et al., Phys. Rev. Lett. 43, 657 (1979).

[52] ZEUS Collaboration, M. Derrick et al., Z. Phys. C63, 391 (1994);

M. Derrick et al., paper pa02-050, submitted to the 28th International Conference on HEP, 25-31 July, 1996, Warsaw, Poland.

[53] W.G. Jones et al., Phys. Rev. Lett. 21, 586 (1968);

C. Berger et al., Phys. Lett. B39, 659 (1972);

SBT Collaboration, J. Ballam et al., Phys. Rev. D5, 545 (1972);

G.E. Gladding et al., ibid. 8, 3721 (1973).

[54] B.Z. Kopeliovich, J. Raufeisen and A.V. Tarasov, Phys. Lett. B440, 151 (1998).

[55] T. Renk, G. Piller and W. Weise, Nucl. Phys. A689, 869 (2001).

[56] J. Hüfner, B.Z. Kopeliovich and A. Zamolodchikov, Z. Phys. A357, 113 (1997).

[57] B.Z. Kopeliovich, J. Nemchik, N.N. Nikolaev and B.G. Zakharov, Phys. Lett. B324, 469 (1994).

[58] A.H. Mueller, in Proc. of the 17th Recontre de Moriond, Moriond. 1982, ed. by J. Tran Thanh Van, p. 13

[59] S.J. Brodsky, in Proc. of the 13th Symposium on Multiparticle Dynamics, ed. by W. Kittel, W. Metzger and A. Stergiou (World Scientific, Singapore, 1982)

[60] B.K. Jennings and B.Z. Kopeliovich, Phys. Rev. Lett. 70, 3384 (1993). 
[61] The E665 Collaboration, M.R. Adams et al., Phys. Rev. Lett. 74, 1525 (1995).

[62] B.Z. Kopeliovich, A.V. Tarasov and O.O. Voskresenskaya, Long-Range Coulomb Forces in DIS: Missed Radiative Corrections?, hep-ph/0105110, to appear in Eur. Phys. J. A.

[63] B.Z. Kopeliovich and J. Nemchik, Where is the Baseline for Color Transparency Studies with Moderate Energy Electron Beams ?, preprint MPIH-V41-1995; nucl-th/9511018.

[64] HERMES Collaboration, K. Ackerstaff et al., Phys. Rev. Lett. 82, 3025 (1999).

[65] A. Borissov, private communication

[66] B.K. Jennings, G.A. Miller, Phys. Rev. D44, 692 (1991).

[67] P.V.R. Murthy et al., Nucl. Phys. B92, 269 (1975).

[68] A. Gsponer et al., Phys. Rev. Lett. 42, 9 (1979).

[69] B.Z. Kopeliovich and J. Nemchik, Phys. Lett. B368, 187 (1996).

[70] L.V. Gribov, E.M. Levin and M.G. Ryskin, Nucl. Phys. B188 (1981) 555; Phys. Rep. 100, $1(1983)$.

[71] B.Z. Kopeliovich, I.K. Potashnikova, B. Povh and E. Predazzi, Phys. Rev. Lett. 85, 507 $(2000)$.

[72] A.H. Mueller and J.W. Qiu, Nucl. Phys. B268, 427 (1986).

[73] B.Z. Kopeliovich, A.V. Tarasov and J. Hüfner, Coherence Phenomena in Charmonium Production off Nuclei at the Energies of RHIC and LHC, hep-ph/0104256, to appear in Nucl. Phys. A. 